\title{
Modulation-Domain Kalman Filtering for Monaural Blind Speech Denoising and Dereverberation
}

\author{
Nikolaos Dionelis, Mike Brookes, Member, IEEE
}

\begin{abstract}
We describe a monaural speech enhancement algorithm based on modulation-domain Kalman filtering to blindly track the time-frequency log-magnitude spectra of speech and reverberation. We propose an adaptive algorithm that performs blind joint denoising and dereverberation, while accounting for the inter-frame speech dynamics, by estimating the posterior distribution of the speech log-magnitude spectrum given the log-magnitude spectrum of the noisy reverberant speech. The Kalman filter update step models the non-linear relations between the speech, noise and reverberation log-spectra. The Kalman filtering algorithm uses a signal model that takes into account the reverberation parameters of the reverberation time, $T_{60}$, and the direct-to-reverberant energy ratio (DRR) and also estimates and tracks the $T_{60}$ and the DRR in every frequency bin to improve the estimation of the speech log-spectrum. The proposed algorithm is evaluated in terms of speech quality, speech intelligibility and dereverberation performance for a range of reverberation parameters and reverberant speech to noise ratios, in different noises, and is also compared to competing denoising and dereverberation techniques. Experimental results using noisy reverberant speech demonstrate the effectiveness of the enhancement algorithm.
\end{abstract}

Index Terms-Speech enhancement, dereverberation, Kalman filtering, minimum mean-square error (MMSE) estimation.

\section{INTRODUCTION}

$\mathrm{N}$ OWADAYS, technology is ever evolving with tremendous haste and the demand for speech enhancement systems is evident. Speech enhancement in noisy reverberant environments, for human listeners, is challenging. Speech is degraded by noise and reverberation when captured using a nearfield or far-field distant microphone [1] [2]. A room impulse response (RIR) can include components at long delays, hence resulting in reverberation and echoes [3] [4]. Reverberation is a convolutive distortion, induces long-term correlation between consecutive observations and can be quite long with a reverberation time, $T_{60}$, of more than $0.8 \mathrm{~s}$. Reverberation and noise, which can be stationary or non-stationary, have a detrimental impact on both speech quality and speech intelligibility.

The direct to reverberant energy ratio (DRR) and the reverberation time, $T_{60}$, are the two main parameters of a reverberation model [5] [1]. The DRR describes reverberation in the space domain, depending on the positions of the source and the receiver. The $T_{60}$ is the time interval required for a sound level to decay $60 \mathrm{~dB}$ after ceasing its original stimulus. The $T_{60}$, when measured in the diffuse sound field, depends on the room and is independent of the source to microphone configuration. The impact of reverberation on perception depends on the $T_{60}$. If the $T_{60}$ is short, the environment reinforces the sound which

N. Dionelis and M. Brookes are with the Department of Electrical and Electronic Engineering, Imperial College London, London SW7 2AZ, U.K. (e-mail: nikolaos.dionelis11@imperial.ac.uk; mike.brookes@imperial.ac.uk). may enhance the sound perception [6] [7]. If the $T_{60}$ is long, then spoken syllables interfere with future ones. Reverberation spreads energy over time and because of this smearing across time, (a) the energy of individual phones spreads out in time, (b) plosives have a delayed decay and fricatives are smoothed, and (c) preceding phones blur into the current phones.

The aim of speech enhancement is to reduce and ideally eliminate the effects of both noise and reverberation without distorting the speech signal [8]. Enhancement algorithms typically aim to suppress noise and late reverberation because early reverberation is not perceived as separate sound sources and usually improves speech quality and intelligibility. Noise is assumed to be uncorrelated with speech, early reverberation is correlated with speech and late reverberation is commonly assumed to be uncorrelated with speech, as in [6] and [9].

Speech enhancement can be performed in different domains. The ideal domain should be chosen such that (a) good models of speech and noise exist in this domain, and (b) speech and noise are separable in this domain. Speech and noise are additive in the Short Time Fourier Transform (STFT) domain [10] [11]. The relation between speech and noise becomes progressively more complicated in the amplitude, power and logpower spectral domains. Noise suppression algorithms operate in a time-frequency domain and these techniques have been extended to address dereverberation. In [9], spectral enhancement methods based on a time-frequency gain, originally developed for noise suppression, have been modified and employed for dereverberation. Such algorithms suppress late reverberation assuming that the early and late reverberation components are uncorrelated. The spectral enhancement methods in [12] [9] estimate the late reverberant spectral variance (LRSV) and use it in the place of the noise spectral variance, reducing the problem of late reverberation suppression to that of estimating the LRSV [6]. In [7], blind spectral weighting is employed to reduce the overlap-masking effect of reverberation using an uncorrelated additive assumption for late reverberation.

Dereverberation methods that leave the phase unaltered and operate in the amplitude, power or log-power spectral domains are relatively insensitive to minor variations in the spatial placement of sources [13]. Two criticisms of spectral enhancement algorithms based on LRSV reverberation noise estimation are that they introduce musical noise and suppress speech onsets when they over-estimate reverberation [14]. The LRSV estimator in [15], which is an extension of [6], models the RIR in the STFT domain and not in the time domain [16], using the RIR model from Polack [17] or Moorer [18] [16]. Reverberation is estimated in [15] using the energy of the direct path of the speech and an external $T_{60}$ estimate.

Modelling the temporal dynamics of speech is beneficial 
when the $T_{60}$ is long and the DRR is low [19] [2]. Blind joint denoising and dereverberation using speech and noise tracking is performed in [19]. The SPENDRED algorithm [20] [21], which is a model-based method with a convolution model for reverberation based on the $T_{60}$ and the DRR, considers the speech temporal dynamics. SPENDRED employs a parametric model of the RIR [22] and performs frequency-dependent and time-varying $T_{60}$ and DRR estimation. However, unless the source or the microphone are moving, the $T_{60}$ and the DRR will be constant throughout the recording. The SPENDRED algorithm assumes that DRR $\geq 1-\left(10^{-6}\right)^{\frac{L}{T_{60}}}$ where $L$ is the acoustic frame increment. For example, when $T_{60}=0.4 \mathrm{~s}$ and $L=5 \mathrm{~ms}$, then DRR $\geq-8 \mathrm{~dB}$ is assumed. In addition, SPENDRED performs intra-frame correlation modelling, which can be beneficial in adverse conditions, while typical algorithms decouple different frequency bands, as in [10].

Statistical-based methods, such as SPENDRED, model reverberation as a convolution in the power spectral domain while LRSV methods treat reverberation as an additive distortion in the power spectral domain [22] [23]. A model with an infinite impulse response is used either with the two parameters of the $T_{60}$ and the DRR, as in [20] [21], or with a larger number of parameters. The low-order convolution model of reverberation with the $T_{60}$ and the DRR is parsimonious [20] [21] and contrasts with the higher-order autoregressive processes in the complex STFT domain used in [24] [25].

The algorithms described in [26], [22] and [23] create nonlinear observation models of noisy reverberant speech in the $\log$ Mel-power spectral domain, using the reverberation-tonoise ratio (RNR). As discussed in [27], phase differences in Mel-frequency bands have different properties from phase differences in STFT bins. The phase factor between reverberant speech and noise is different from that between speech and noise and is examined in [22] for Mel-frequency bands.

In noisy reverberant conditions, identifying speech onsets and determining which frames are unvoiced/silence is difficult, due to the smearing across time that leads to noise over-estimation. The concatenation of different techniques for denoising and dereverberation was found in [19] to have lower performance than unified methods due to over-estimating noise when estimating noise and reverberation separately. Nevertheless, long-term linear prediction with pre-denoising can be used to suppress noise and reverberation. In the weighted prediction error (WPE) algorithm [28] [2], reverberation is modelled by a one-dimensional convolution in each frequency bin. In [29], the WPE method is discussed along with interframe correlation. In [30], the WPE method is used in the complex STFT domain performing batch processing and iteratively estimating, first, the reverberation prediction coefficients and, then, the speech spectral variance. In [31] [32], WPE linear filtering is employed in the STFT power spectral domain.

In recent years, much effort has been devoted to developing speech enhancement and dereverberation algorithms that use deep learning (DL) approaches to estimate either the STFT amplitudes directly or, more commonly, a real or complex valued gain mask in the STFT domain [33]. These DL approaches, such as the ones presented in [34], [35] and [36], have consistently been shown to give enhancement performance that is better than conventional model-based enhancement, such as the Log-MMSE enhancer [37], especially in highly non-stationary noise. Data-driven methods have also been applied successfully to removing both noise and reverberation either jointly, in [38] and [39], or sequentially, in [40] and [41]. Dereverberation based on supervised learning using ratio or binary masking [42], considering the early and late reverberant speech components, is examined in [43]. A concern with DL approaches to enhancement and dereverberation has been a lack of robustness to mismatches between the type of noise in training and testing. It has been observed that speech enhancement performance declines in mismatched conditions [44] and a number of researchers have tried to improve robustness by including model-based features as additional inputs [45] [46]. In DL approaches, there is also an important computational aspect to be considered. Each language and combination of environmental factors requires a separate round of training resulting in an exponential complexity burden.

The use of a time-domain Kalman filter (KF) for speech enhancement was first suggested by [47]. This was extended by [48] who proposed tracking both speech and noise and proposed two alternative methods of estimating time-dependent AR models for the speech and noise. However, it was found that neither method outperformed the Log-MMSE enhancer [37]. In [49], a KF enhancer was combined with a perceptual post-filter based on the masking properties of the human ear. In [50] [51], a modulation-domain speech enhancer was proposed that used a KF to estimate the clean speech spectral amplitudes using a linear KF update step and this was found to outperform the time-domain KF of [47]. This approach was extended in [10] to use an improved signal model in which speech and noise were additive in the complex STFT domain rather than in the spectral amplitude domain. This was further developed in [52] to minimise the squared error in the $\log$ spectral domain and to track the speech phase in addition to the speech and noise amplitudes. Non-linear KFs that take into account that speech and noise add in the STFT domain are formulated in [53], [54] and [11]. A modulation domain KF proposed in [55] used a complex AR model of order 20, which corresponds to $320 \mathrm{~ms}$, for dereverberation. It used a similar approach to that of [48] to estimate both the speech spectral values and the AR coefficients online. In contrast to traditional KFs that are linear, do not model reverberation and are implemented in the time domain [47], the proposed algorithm is non-linear, performs blind speech dereverberation and models reverberation as an autoregressive process in the prediction step and as a phase-sensitive process in the update step. In this paper, phase-sensitive modulation-domain Kalman filtering for speech enhancement [56], joint noise suppression and dereverberation, is performed in an adaptive manner.

This paper presents an adaptive denoising and dereverberation Kalman filtering framework that tracks the speech and reverberation spectral log-magnitudes. In this paper, we extend the enhancer in [53] to include dereverberation, taking into account inter-frame correlations. We use an integrated structure of two parallel signal models to track speech, reverberation and the $T_{60}$ and DRR reverberation parameters. The $T_{60}$ and the DRR are updated in every frame to improve the estimation 
of the speech log-spectrum. We create an observation model and a series of non-linear KF update steps that perform joint noise and reverberation suppression by estimating the first two moments of the posterior of the speech log-spectrum given the noisy reverberant log-spectrum. In contrast to [50] and [10], the log-spectral domain is chosen for the KF state, as in [52] and [53], because speech log-spectra are well modelled by Gaussian distributions and the mean squared error (MSE) in the log-spectral domain is a good measure to use for perceptual quality [57] [58]. Modelling spectral log-amplitudes as Gaussian distributions leads to good speech modelling in noisy reverberant environments because super-Gaussian distributions can model the speech amplitude spectrum well [11] [54].

The structure of this paper is as follows. Section II describes the signal model and Sec. III presents the enhancement algorithm and its non-linear KF. The implementation and the validation of the algorithm are in Sec. IV. The algorithm's evaluation is in Sec. V. Conclusions are drawn in Sec. VI.

\section{SignAL MODEL AND NOTATION}

In the complex STFT domain, the noisy speech, $Y_{t}(k)$, is given by $Y_{t}(k)=S_{t}(k)+R_{t}(k)+N_{t}(k)$ where $S_{t}(k)$ is the direct speech component, $R_{t}(k)$ is the reverberant speech component and $N_{t}(k)$ is the noise, as for example in [24] [25]. The time-frame index is $t$ and the frequency bin index is $k$. For convenience, we define $Z_{t}(k)=R_{t}(k)+N_{t}(k)$. We drop the time and frequency indexes and we obtain $Y=S+Z=$ $S+R+N$. We define the log-magnitude spectrum of $S$ as $s=\log (|S|)$ and we also define $r, n, y$ and $z$ similarly.

The algorithm treats each frequency bin on its own. Within the algorithm, $t$ is included as a subscript in equations involving multiple frames. Signal quantities with capital letters, such as $S_{t}$, are complex numbers with magnitude and phase, $\left|S_{t}\right|$ and $\angle S_{t}$. The reverberation power, $\left|R_{t}\right|^{2}$, in frame $t$ consists of two components: (a) the decaying reverberation that was present in the previous frame with power $a_{t}\left|R_{t-1}\right|^{2}$, and (b) a new reverberation component arising from the speech signal in the previous frame with power $b_{t}\left|S_{t-1}\right|^{2}$. Here, $a_{t}, b_{t} \in \mathbb{R}$ respectively model the decay rate of the old reverberation component and the scale factor of the new reverberation component. The reverberation signal model is given by

$$
\begin{aligned}
R_{t} & =\sqrt{a_{t}} R_{t-1} \exp \left(j \theta_{t}\right)+\sqrt{b_{t}} S_{t-1} \exp \left(j \psi_{t}\right) \\
& =\sum_{\tau=1}^{\infty}\left(\prod_{i=1}^{\tau-1}\left(\sqrt{a_{t-i+1}} \exp \left(j \theta_{t-i+1}\right)\right)\right. \\
& \left.\times \sqrt{b_{t-\tau+1}} S_{t-\tau} \exp \left(j \psi_{t-\tau+1}\right)\right) .
\end{aligned}
$$

If both $a_{t}$ and $b_{t}$ are time-invariant and $S_{t}$ is a unit impulse at $t=0$, then (1) reduces to $\left|R_{t}\right|^{2}=b_{t} a_{t}^{t-1}$ with a total reverberation power of $\sum_{t=1}^{\infty}\left(\left|R_{t}\right|^{2}\right)=\frac{b_{t}}{1-a_{t}}$ from which

$$
a_{t}^{\frac{T_{60}}{L}}=10^{-6}, \quad b_{t}=\frac{1-a_{t}}{\mathrm{DRR}}
$$

where $L$ is the acoustic frame increment in seconds. In (1), the factors $\exp \left(j \theta_{t}\right)$ and $\exp \left(j \psi_{t}\right)$, where $\theta_{t}$ and $\psi_{t}$ are assumed to be uniformly distributed phases, denote the phases of the old and new reverberation components, respectively. In (2),
Table I

List of the main variables in the log-magnitude spectral domain

\begin{tabular}{|c|c|c|}
\hline Variable & Equation & Description \\
\hline$n_{t}$ & $\log \left(\left|N_{t}\right|\right)$ & Noise signal \\
\hline$r_{t}$ & $\log \left(\left|R_{t}\right|\right)$ & Reverberation signal \\
\hline$s_{t}$ & $\log \left(\left|S_{t}\right|\right)$ & Speech signal \\
\hline$y_{t}$ & $\log \left(\left|Y_{t}\right|\right)$ & Noisy reverberant speech signal \\
\hline$z_{t}$ & $\log \left(\left|Z_{t}\right|\right)$ & Reverberation plus noise signal \\
\hline$\beta_{t}$ & $(5)$ & Reverberation scale factor \\
\hline$\gamma_{t}$ & $(4)$ & Reverberation decay factor \\
\hline$\delta_{t}$ & $(4)$ & Old reverberation component \\
\hline$\epsilon_{t}$ & $(5)$ & New reverberation component \\
\hline
\end{tabular}

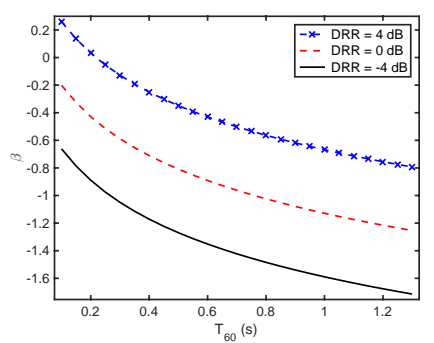

(a)

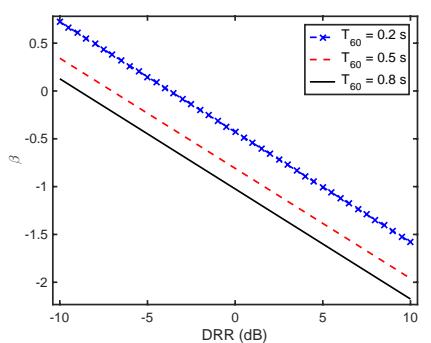

(b)
Figure 1. Plot of $\beta$, using $L=8 \mathrm{~ms}$ in (2), against: (a) $T_{60}$ when the DRR is 4,0 and $-4 \mathrm{~dB}$, and (b) DRR when the $T_{60}$ is $0.2,0.5$ and $0.8 \mathrm{~s}$.

the DRR is defined in the power spectral domain [20] [21]. The $T_{60}$ and the DRR depend on time and frequency [5].

Equation (1) is the convolution model for reverberation; the most common reverberation model is this single-pole filter described by the pole and zero positions that depend on the $T_{60}$ and the DRR [20] [2]. A convolution of infinite order is used in (1) to describe reverberation [20] [21], and models that describe reverberation by a convolution are also discussed in [22] [23]. The signal model is defined by (1) and by

$$
\begin{array}{ll}
Y_{t}=S_{t}+Z_{t}, & Z_{t}=R_{t}+N_{t}, \\
\gamma_{t}=0.5 \log \left(a_{t}\right), & \delta_{t}=\gamma_{t}+r_{t-1}=\log \left(\sqrt{a_{t}}\left|R_{t-1}\right|\right), \\
\beta_{t}=0.5 \log \left(b_{t}\right), & \epsilon_{t}=\beta_{t}+s_{t-1}=\log \left(\sqrt{b_{t}}\left|S_{t-1}\right|\right)
\end{array}
$$

where $b_{t}>0,0<a_{t}<1$ and $\gamma_{t}<0$. A list of variables in the log-magnitude spectral domain is shown in Table I.

Figure 1 shows graphs of $\beta$ against $T_{60}$ for a fixed DRR and of $\beta$ against DRR for a fixed $T_{60}$. If DRR $=0 \mathrm{~dB}$, then $b_{t}=1-a_{t}$. If $\beta_{t}=0$, then $b_{t}=1$ and DRR $=1-a_{t}$.

Figure 2 depicts the flowchart of the signal model. Equations (1) and (3), together with Fig. 2, define the high-level signal decomposition. The reverberation signal model in (1) uses $\sqrt{a_{t}}$ and $\sqrt{b_{t}}$ because the $a_{t}$ and $b_{t}$ reverberation parameters, in (2), are defined in the power spectral domain [20] [21]. The $a_{t}$ and $b_{t}$ parameters are mapped to $\gamma_{t}$ and $\beta_{t}$ using (4) and (5). The signals $z_{t}, \delta_{t}$ and $\epsilon_{t}$ are the total distubance, the old (decaying) reverberation and the new reverberation, respectively. In this case, $z_{t}$ is defined in Table I and $\delta_{t}$ and $\epsilon_{t}$ in (4) and (5).

The signal model of how the reverberation parameters of $\gamma_{t}$ and $\beta_{t}$ change over time is a random walk model. This is used in the algorithm's KF prediction step for $\gamma_{t}$ and $\beta_{t}$.

The signal model in Fig. 2 is linked to the alternating and interacting KFs of the algorithm. The algorithm is a collection 


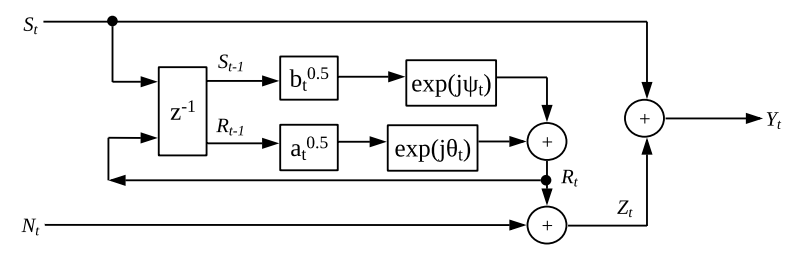

Figure 2. The flowchart diagram of the proposed signal model of the speech enhancement algorithm. The KF's signal model is given by (1) and (3).

of two KFs, the speech KF and the reverberation KF, that estimate the speech and reverberation log-spectra and the $\gamma_{t}$ and $\beta_{t}$ reverberation parameters, as described in Sec. III.

\section{THE SPEECH ENHANCEMENT ALGORITHM}

The adaptive KF algorithm operates in the log-magnitude spectral domain and Fig. 3 depicts the denoising and dereverberation algorithm that formulates a model of reverberation as a first-order autoregressive process. The algorithm propagates the means and variances of the random variables. Almost all the signals are assumed to follow a Gaussian distribution and the distribution of $s_{t}$ conditioned on observations up to time $\tau$ is denoted by $p_{s_{t \mid \tau}}(s) \triangleq p\left(s_{t} \mid y_{0}, \ldots, y_{\tau}\right)=N\left(s_{t \mid \tau}, \Sigma_{t \mid \tau}^{(s)}\right)$. A Gaussian distribution is denoted by its mean, $s_{t \mid \tau}$.

The core of the algorithm in Fig. 3 is the KF that comprises gray blocks in the flowchart diagram. The non-linear KF estimates and tracks the posterior distributions of the speech log-magnitude spectrum, $s_{t}$, the reverberation log-magnitude spectrum, $r_{t}$, and the reverberation parameters, $\gamma_{t}$ and $\beta_{t}$.

The input to the algorithm in Fig. 3 is the noisy reverberant speech in the time domain. The algorithm's first step is to obtain the signal in the complex STFT domain. The algorithm does not alter the noisy reverberant phase, $\angle Y$, and uses the noisy reverberant amplitude spectrum, $|Y|$, in three ways: in the speech KF prediction step, in the KF update step and in the noise power modelling. The speech $\mathrm{KF}$ state, $\mathbf{s}_{t} \in \mathbb{R}^{p}$, is the speech log-spectrum from the previous $p$ frames [52],

$$
\mathbf{s}_{t}=\left(\begin{array}{llll}
s_{t} & s_{t-1} & \ldots & s_{t-p+1}
\end{array}\right)^{T} .
$$

The linear speech KF prediction step is based on autoregressive (AR) modelling on the log-spectrum of pre-cleaned speech. The reverberation KF state is $r_{t}$ and the KF states of the reverberation parameters are $\gamma_{t}$ and $\beta_{t}$. The KF observation is the noisy reverberant speech log-spectrum, $y_{t}$, which is used in the KF update step to compute the first two moments of the posterior of the speech log-spectrum. The mean of the speech log-spectrum posterior is used together with $\angle Y$ to create the enhanced speech signal using the inverse STFT (ISTFT).

Apart from the speech log-spectrum, the non-linear KF also tracks the reverberation log-spectrum, $r_{t}$, and the $\gamma_{t}$ and $\beta_{t}$ reverberation parameters. Hence, the KF state is of dimension $(p+3)$ and may be partitioned as $\left(\mathbf{s}_{t}^{T} r_{t} \gamma_{t} \beta_{t}\right)^{T}$. The KF, as defined by the gray blocks in Fig. 3, has a speech KF prediction step, a reverberation KF prediction step and a series of KF update steps. The reverberation KF is comprised of the blocks "Reverberation KF prediction", "KF Update" and " $\gamma_{t}$, $\beta_{t}$ KF Update". These three blocks perform joint denoising and dereverberation and also estimate both $s_{t \mid t}$ and $\Sigma_{t \mid t}^{(\mathrm{s})}$.
The structure of this section is as follows. Sections III.A and III.B present the speech and reverberation prediction steps, respectively. Sections III.C and III.D describe the update step and Sec. III.E the priors for the $\gamma_{t}$ and $\beta_{t}$ parameters that are needed so that the KF (a) distinguishes between $s_{t}$ and $r_{t}$, and (b) does not diverge to non-realistic $\gamma_{t}$ and $\beta_{t}$ estimates. Finally, Sec. III.F presents the unshaded blocks in Fig. 3.

\section{A. The Speech KF Prediction Step}

The purpose of the speech KF prediction step is to predict the prior of the state vector, $s_{t \mid t-1}$, from the previous frames posterior distribution, $s_{t-1 \mid t-1}$, [52]. The AR modelling and the KF prediction step correlate the current frame element of the speech state vector, $\mathbf{s}_{t}$, with the previous $(p-1)$ frame elements of $\mathbf{s}_{t}$ [53]. For $\mathbf{s}_{t}$, the speech signal model is

$$
\mathbf{s}_{t \mid t-1}=\mathbf{A}_{t}^{(\mathrm{s})}\left(\mathbf{s}_{t-1 \mid t-1}-\zeta_{t}^{(\mathrm{s})} \mathbf{1}_{p}\right)+\zeta_{t}^{(\mathrm{s})} \mathbf{1}_{p}+\mathbf{w}_{t}^{(\mathrm{s})}
$$

where $\mathbf{1}_{p}$ denotes the unity vector, $\mathbf{1}_{p} \in \mathbb{R}^{p}$. In (7), $\zeta_{t}^{(\mathrm{s})} \in \mathbb{R}$ is the AR mean [52], $\mathbf{w}_{t}^{(\mathrm{s})} \in \mathbb{R}^{p}$ with only its first element being non-zero is zero-mean with covariance matrix $\mathbf{Q}_{t}^{(\mathrm{s})}$, and

$$
\begin{aligned}
& \mathbf{A}_{t}^{(\mathrm{s})}=\left(\begin{array}{c}
-\mathbf{a}_{t}^{(\mathrm{s}) T} \\
\mathbf{I}_{(p-1)} \\
\mathbf{0}_{(p-1) \times 1}
\end{array}\right) \in \mathbb{R}^{p \times p}, \\
& \mathbf{Q}_{t}^{(\mathrm{s})}=\mathbb{E}\left\{\mathbf{w}_{t}^{(\mathrm{s})} \mathbf{w}_{t}^{(\mathrm{s}) T}\right\}=\left(\begin{array}{cl}
\epsilon_{t}^{(\mathrm{s})} & \mathbf{0}_{(p-1) \times 1}^{T} \\
\mathbf{0}_{(p-1) \times 1} & \mathbf{0}_{(p-1) \times(p-1)}
\end{array}\right)
\end{aligned}
$$

where $\mathbf{I}_{p} \in \mathbb{R}^{p \times p}$ denotes the identity matrix and $\mathbf{0}_{p \times p} \in$ $\mathbb{R}^{p \times p}$ the zero matrix. The $\mathrm{KF}$ transition matrix is $\mathbf{A}_{t}^{(\mathrm{s})}$, the $\mathrm{KF}$ transition noise covariance matrix is $\mathbf{Q}_{t}^{(\mathrm{s})}$ and the $\mathrm{KF}$ transition noise is $\mathbf{w}_{t}^{(\mathrm{s})}$. The AR coefficients vector is $\mathbf{a}_{t}^{(\mathrm{s})} \in \mathbb{R}^{p}$ and the variance of the transition noise is $\epsilon_{t}^{(\mathrm{s})}$. In (7), $\mathbf{A}_{t}^{(\mathrm{s})}, \zeta_{t}^{(\mathrm{s})}$ and $\mathbf{Q}_{t}^{(\mathrm{s})}$ are estimated from AR modeling of order $p$, i.e. $\operatorname{AR}(p)$ modeling. The AR mean, $\zeta_{t}^{(\mathrm{s})}$, is the mean speech spectral log-amplitude estimated as an AR parameter, as in [52].

The KF prediction step, which uses conditional distributions to model short-term dependencies [52] [53], comprises the blocks: "Speech KF prediction", "Decorrelate" and "Recorrelate". Decorrelation and recorrelation of $\mathbf{s}_{t}$ are performed after and before the KF prediction, respectively. Decorrelation and recorrelation are performed so that the non-linear update step can be applied and perform vector-matrix and matrix-matrix multiplications for the speech KF state mean and its covariance matrix, respectively, using $\mathbf{B}_{t} \in \mathbb{R}^{p \times p}$ [54]. The outputs of the "Decorrelate" block are $s_{t}$ and the rest elements of $\mathbf{s}_{t}$.

The prediction step propagates the first two moments of the state [52] [58] and predicts $\mathbf{s}_{t}$ as the linear transformation of $\mathbf{s}_{t-1}$ given in (7) using the AR coefficients obtained from the "Speech AR(p)" block in Fig. 3, which has pre-cleaned speech as an input. After the prediction step, $\mathbf{s}_{t}$ is correlated so in order to simplify the KF update and impose the observation constraint [10], we apply a linear transformation matrix, $\mathbf{B}_{t}$. We decorrelate $\mathbf{s}_{t}$ with a linear transformation and $\mathbf{B}_{t}$ is chosen to decorrelate the current frame element, $s_{t}$, from the rest of the state vector so that the algorithm performs the update step with the variable in the current frame [54]. After the KF update step, recorrelation is applied with a linear transformation, using $\mathbf{B}_{t}^{-1}$, to continue the KF recursion and to preserve the inter-frame correlation created from the prediction step. 


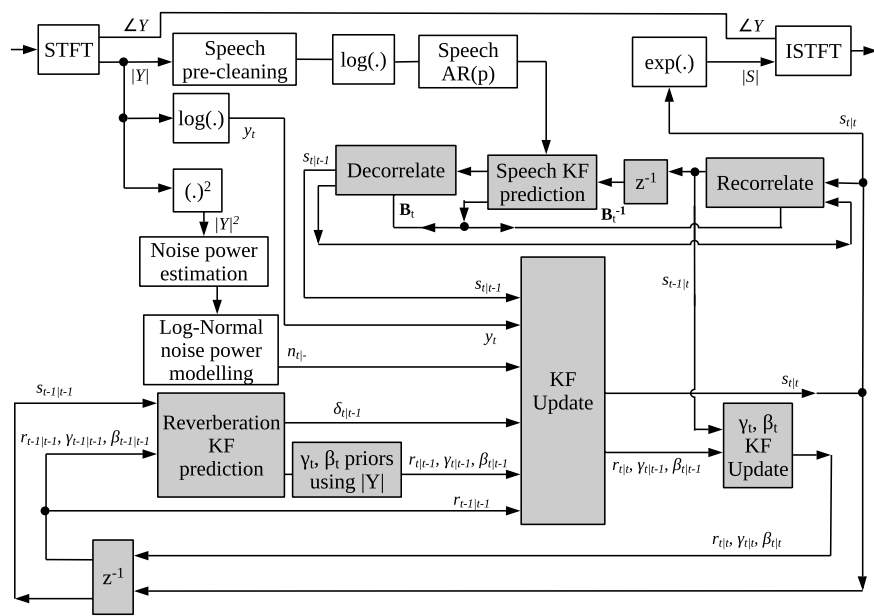

Figure 3. The flowchart of the algorithm to suppress noise and reverberation For clarity, the signal variances are not included. The gray blocks constitute the $\mathrm{KF}$ that tracks the speech and reverberation log-spectra and the reverberation parameters. The "KF Update" and " $\gamma_{t}, \beta_{t}$ KF Update" blocks constitute the non-linear KF update step that uses the signal model described in Sec. II.

A bar diacritic,,- , is used to indicate the transformed domain, $\overline{\mathbf{s}}=\mathbf{B s}$. The posteriors, $\overline{\mathbf{s}}_{t \mid t}$ and $\overline{\boldsymbol{\Sigma}}_{t \mid t}^{(\mathrm{s})}$, are computed in the KF update step in this domain. First, $\boldsymbol{\Sigma}_{t \mid t-1}^{(\mathrm{s})}$ is partitioned as

$$
\Sigma_{t \mid t-1}^{(\mathrm{s})}=\left(\begin{array}{rr}
\Sigma_{t \mid t-1}^{(\mathrm{s})} & \mathbf{p}_{B, t \mid t-1}^{T} \\
\mathbf{p}_{B, t \mid t-1} & \mathbf{P}_{C, t \mid t-1}
\end{array}\right)
$$

where a sufficient condition for $\Sigma_{t \mid t-1}^{(\mathrm{s})}$ to be non-zero is that $\epsilon_{t}^{(\mathrm{s})}$ in (9) is non-zero. For decorrelation, $\mathbf{B}_{t}$ is given by

$$
\mathbf{B}_{t}=\left(\begin{array}{crr}
1 & & \mathbf{0}_{1 \times(p-1)} \\
-\left(\Sigma_{t \mid t-1}^{(\mathrm{s})}\right)^{-1} & \mathbf{p}_{B, t \mid t-1} & \mathbf{I}_{(p-1)}
\end{array}\right) .
$$

The transformed mean and covariance matrix are given by

$$
\begin{aligned}
& \overline{\mathbf{s}}_{t \mid t-1}=\mathbf{B}_{t} \mathbf{s}_{t \mid t-1}=\left(s_{t \mid t-1} \overline{\mathbf{s}}_{C, t \mid t-1}^{T}\right)^{T}, \\
& \overline{\boldsymbol{\Sigma}}_{t \mid t-1}^{(\mathrm{s})}=\mathbf{B}_{t} \boldsymbol{\Sigma}_{t \mid t-1}^{(\mathrm{s})} \mathbf{B}_{t}^{T}=\left(\begin{array}{cc}
\Sigma_{t \mid t-1}^{(\mathrm{s})} & \mathbf{0}_{1 \times(p-1)} \\
\mathbf{0}_{(p-1) \times 1} & \overline{\mathbf{P}}_{\mathrm{C}, t \mid t-1}
\end{array}\right) \\
& \overline{\mathbf{P}}_{\mathrm{C}, t \mid t-1}=\mathbf{P}_{C, t \mid t-1}-\left(\Sigma_{t \mid t-1}^{(\mathrm{s})}\right)^{-1} \mathbf{p}_{B, t \mid t-1} \mathbf{p}_{B, t \mid t-1}^{T}
\end{aligned}
$$

where $\Sigma_{t \mid t-1}^{(\mathrm{s})}$ is preserved as the leading element in (13) and $\overline{\mathbf{s}}_{\mathrm{C}, t \mid t-1} \in \mathbb{R}^{(p-1)}$ contains the elements that will not be changed by the non-linear update step in Sec. III.C. After the update step, the inverse transformation is applied with

$$
\mathbf{s}_{t \mid t}=\mathbf{B}_{t}^{-1} \overline{\mathbf{s}}_{t \mid t}, \quad \boldsymbol{\Sigma}_{t \mid t}^{(\mathrm{s})}=\mathbf{B}_{t}^{-1} \overline{\boldsymbol{\Sigma}}_{t \mid t}^{(\mathrm{s})} \mathbf{B}_{t}^{-T} .
$$

The posteriors $\overline{\mathbf{s}}_{t \mid t}$ and $\overline{\boldsymbol{\Sigma}}_{t \mid t}^{(\mathrm{s})}$ are found and $\overline{\mathbf{s}}_{t \mid t}$ is given by

$$
\overline{\mathbf{s}}_{t \mid t}=\left(s_{t \mid t} \overline{\mathbf{s}}_{C, t \mid t}^{T}\right)^{T} \quad \text { where } \overline{\mathbf{s}}_{C, t \mid t}=\overline{\mathbf{s}}_{C, t \mid t-1} .
$$

Using $\overline{\mathbf{P}}_{C, t \mid t}=\overline{\mathbf{P}}_{C, t \mid t-1}$, the posterior covariance matrix, $\overline{\boldsymbol{\Sigma}}_{t \mid t}^{(\mathrm{s})}$, is obtained by replacing $\Sigma_{t \mid t-1}^{(\mathrm{s})}$ in (13) by $\Sigma_{t \mid t}^{(\mathrm{s})}$. Hence,

$$
\begin{aligned}
\bar{\Sigma}_{t \mid t}^{(\mathrm{s})} & =\left(\begin{array}{cc}
\Sigma_{t \mid t}^{(\mathrm{s})} & \mathbf{0}_{1 \times(p-1)} \\
\mathbf{0}_{(p-1) \times 1} & \overline{\mathbf{P}}_{C, t \mid t}
\end{array}\right) \\
\Sigma_{t \mid t}^{(\mathrm{s})} & =\mathbb{E}\left\{s_{t}^{2} \mid \mathcal{Y}_{t}\right\}-\left(\mathbb{E}\left\{s_{t} \mid \mathcal{Y}_{t}\right\}\right)^{2}
\end{aligned}
$$

where $\mathcal{Y}_{t}=\left\{y_{0}, y_{1}, \ldots, y_{t}\right\}$. In the update step in Sec. III.C, $\mathbb{E}\left\{s_{t} \mid \mathcal{Y}_{t}\right\}$ and $\mathbb{E}\left\{s_{t}^{2} \mid \mathcal{Y}_{t}\right\}$ are computed. This formulation allows the use of non-Gaussian priors and is equivalent to the conventional KF if the state is assumed Gaussian [10].

\section{B. The Reverberation KF Prediction Step}

The algorithm uses a random-walk KF prediction step for $\gamma_{t}$ and $\beta_{t}$ that assumes that their means are preserved and their variances increase. A random-walk prediction step was used in [24]. The KF prediction step for $\gamma_{t}$ and $\beta_{t}$ is given by

$$
\begin{array}{ll}
\gamma_{t \mid t-1}^{\prime}=\gamma_{t-1 \mid t-1}, & \Sigma_{t \mid t-1}^{\left(\gamma^{\prime}\right)}=\Sigma_{t-1 \mid t-1}^{(\gamma)}+Q_{\gamma}, \\
\beta_{t \mid t-1}^{\prime}=\beta_{t-1 \mid t-1}, & \Sigma_{t \mid t-1}^{\left(\beta^{\prime}\right)}=\Sigma_{t-1 \mid t-1}^{(\beta)}+Q_{\beta}
\end{array}
$$

in which $Q_{\gamma}$ and $Q_{\beta}$ are fixed variance increments for $\gamma_{t}$ and $\beta_{t}$ whose values depend on the expected rate of change of the $T_{60}$ and DRR parameters, as discussed in Sec. IV. The variances of the reverberation parameter distributions will always increase in the KF prediction step but will normally then decrease in the update step described in Sec. III.C.

After the reverberation KF prediction step, the algorithm computes and imposes priors on $\gamma_{t}$ and $\beta_{t}$ using GaussianGaussian multiplication. These internally computed priors in the " $\gamma_{t}, \beta_{t}$ priors" block in Fig. 3 are described in Sec. III.E. A prime diacritic, I, is used in (19) and (20) to denote the signals before the priors are imposed. After imposing the priors in Sec. III.E, we obtain $\gamma_{t \mid t-1}, \Sigma_{t \mid t-1}^{(\gamma)}$ and $\beta_{t \mid t-1}, \Sigma_{t \mid t-1}^{(\beta)}$.

The "Reverberation KF prediction" block estimates the first two moments of the prior distribution of the reverberation spectral log-amplitude, i.e. $r_{t \mid t-1}$ and its variance. The algorithm performs a reverberation KF prediction step based on the previous posterior of both speech and reverberation using the signal model in (1), where $a$ is less than unity and this ensures that the reverberation KF prediction step is stable.

From (1) and Fig. 2, the STFT-domain reverberation is the sum of two components arising, respectively, from the reverberation and speech components of the previous frame. The old reverberation, $\delta_{t}$, and the new reverberation, $\epsilon_{t}$, are defined in (4) and (5), respectively. The algorithm calculates the prior distributions of these components in the log-spectral domain using $\delta_{t \mid t-1}=\gamma_{t \mid t-1}+r_{t-1 \mid t-1}$ and $\epsilon_{t \mid t-1}=\beta_{t \mid t-1}+s_{t-1 \mid t-1}$. These two equations are based on (4) and (5) with a common condition added to all terms. Assuming that $r_{t-1}$ and $s_{t-1}$ are uncorrelated with $\gamma_{t}$ and $\beta_{t}$, respectively, the variances of the two Gaussian distributions add, in each case, to give

$$
\begin{aligned}
& \Sigma_{t \mid t-1}^{(\delta)}=\Sigma_{t \mid t-1}^{(\gamma)}+\Sigma_{t-1 \mid t-1}^{(\mathrm{r})}, \\
& \Sigma_{t \mid t-1}^{(\epsilon)}=\Sigma_{t \mid t-1}^{(\beta)}+\Sigma_{t-1 \mid t-1}^{(\mathrm{s})} .
\end{aligned}
$$

The "Reverberation KF prediction" block in Fig. 3 computes $r_{t \mid t-1}$. The reverberation log-amplitude spectrum is estimated by modelling the addition in the complex STFT domain of two random variables in the log-amplitude spectral domain. Given two disturbance sources, i.e. $\delta_{t}$ and $\epsilon_{t}$, the blind $\mathrm{KF}$ algorithm combines them into a single disturbance source, i.e. $r_{t}$.

For the remainder of Sec. III.B, the time subscript, $t \mid t-1$, is omitted for clarity. For example, $p(\delta)$ is used instead of $p_{\delta_{t \mid t-1}}(\delta)$ to denote the probability distribution of $\delta_{t \mid t-1}$. 
From (1), the reverberation component is the STFT-domain sum of two elements arising, respectively, from the reverberation and speech components in the previous frame. The log-amplitude spectral domain distributions of these two elements, $\delta_{t \mid t-1}$ and $\epsilon_{t \mid t-1}$, were calculated in the preceding paragraphs. A two-dimensional Gaussian distribution is used for $p(\delta, \epsilon)=p(\delta) p(\epsilon)$ assuming independence between $\delta$ and $\epsilon$. We assume that the phase difference, $\eta=\theta-\psi$, between the two disturbance sources in (1) is uniformly distributed, i.e. $\eta \sim U(-\pi, \pi)$, and independent of their magnitudes. Multiplying (1) by its complex conjugate, taking logs and applying (4) and (5) leads to $e^{2 r}=e^{2 \delta}+e^{2 \epsilon}+2 \cos (\eta) e^{\delta+\epsilon}$ from which $r=0.5 \log \left(e^{2 \delta}+e^{2 \epsilon}+2 \cos (\eta) e^{\delta+\epsilon}\right)$ that depends on $\eta$, as in [52], [53] and [58]. Next, the KF algorithm calculates

$$
\begin{aligned}
\mathbb{E}\{r\} & =\int_{\eta=-\pi}^{\pi} p(\eta) \int_{\delta, \epsilon} r p(\delta, \epsilon) d \delta d \epsilon d \eta \\
& =\int_{\eta=0}^{\pi} \frac{1}{\pi} \int_{\delta, \epsilon} 0.5 \log \left(e^{2 \delta}+e^{2 \epsilon}+2 \cos (\eta) e^{\delta+\epsilon}\right) \\
& \times p(\delta) p(\epsilon) d \delta d \epsilon d \eta, \\
\mathbb{E}\left\{r^{2}\right\} & =\int_{\eta=0}^{\pi} \frac{1}{\pi} \int_{\delta, \epsilon}\left(0 . 5 \operatorname { l o g } \left(e^{2 \delta}+e^{2 \epsilon}\right.\right. \\
& \left.\left.+2 \cos (\eta) e^{\delta+\epsilon}\right)\right)^{2} \times p(\delta) p(\epsilon) d \delta d \epsilon d \eta .
\end{aligned}
$$

The first two moments of $r_{t \mid t-1}$ are estimated with (23) and (24). Here, $K_{(\delta, \epsilon)}$ sigma points are used to evaluate the inner integral over $(\delta, \epsilon)$ and $K_{\eta}$ the outer integral over $\eta$ [59].

\section{The Non-Linear KF Update Step}

The KF algorithm decomposes the noisy reverberant observation, $y_{t}$, into its component parts using distributions in the log-magnitude spectral domain. The decompositions are based on Fig. 2 and the signal model in (3) and (1) which allows the algorithm to perform a series of low-dimensional operations instead of a single high-dimensional one. The KF algorithm propagates backwards through Fig. 2 decomposing: (a) $y_{t}$ into speech, $s_{t}$, and reverberation plus noise, $z_{t}$, (b) the reverberation plus noise, $z_{t}$, into reverberation, $r_{t}$, and noise, $n_{t}$, and (c) the reverberation, $r_{t}$, into "old reverberation", $\delta_{t}$, and "new reverberation", $\epsilon_{t}$. The reverberation plus noise, $z_{t}$, is a variable of the "KF Update" block shown in Fig. 3.

The KF algorithm in Fig. 3 uses $y_{t}$ to first update $s_{t}$ and $z_{t}$ and then to update $r_{t}$. The posterior $z_{t \mid t}$ is computed in the "KF Update" block in Fig. 3. In the algorithm, the observed $y_{t}$ affects $z_{t \mid t}$ directly and, in turn, $z_{t \mid t}$ affects $r_{t \mid t}$. We hence divide the observation update into two steps: (a) we use the log-spectrum observation, $y_{t}$, to estimate the posterior distributions $s_{t \mid t}$ and $z_{t \mid t}$ because $Y_{t}=S_{t}+Z_{t}$ in (3), and (b) we use $z_{t \mid t}$ as an "observation" to obtain the posterior distributions $r_{t \mid t}$ and $n_{t \mid t}$ because $Z_{t}=R_{t}+N_{t}$ in (3). In (b), we calculate an updated version of $r_{t}$ by using the posterior $z_{t \mid t}$ as a KF observation constraint. According to (a) and (b), observing $y_{t}$ provides new information about $s_{t}$ and $r_{t}$.

The sequence of operations involved in the "Reverberation KF prediction", "KF update" and " $\gamma_{t}, \beta_{t}$ KF update" blocks are listed in Table II. The "Reverberation KF prediction" block in Fig. 3, which was presented in Sec. III.B, performs the first five operations. The "KF update" and " $\gamma_{t}, \beta_{t} \mathrm{KF}$ update" blocks perform the next seven operations, i.e. steps $6-12$, and the " $z^{-1}$ " block performs step 13. These 13 steps constitute the dereverberation update step that computes the first two moments of the posterior distributions for most signal quantities and, moreover, includes the prediction step as well for some signal quantities. The means and variances of the tracked Gaussian signals are computed. For clarity, variances, such as $\Sigma_{t \mid t}^{(\mathrm{s})}$ for $s_{t \mid t}$ [52], are not included in Table II.

Table II

The operations performed in the "Reverberation KF prediction", "KF Update" and " $\gamma_{t}, \beta_{t}$ KF Update" blocks in Fig. 3. Steps 7-12 perform specific signal decompositions propagating backwards through Fig. 2. Within the KF loop, the gray blocks in Fig. 3 track the signals $s_{t}, r_{t}, \gamma_{t}$ and $\beta_{t}$.

\begin{tabular}{ll}
\hline & $\begin{array}{l}\text { Inputs: (a) } s_{t \mid t-1} \text { from the speech KF prediction step, (b) } \\
\\
n_{t \mid-} \text { from external noise estimation, (c) } y_{t} \text { from observation, } \\
\text { and (d) } s_{t-1 \mid t} \text { from } s_{t \mid t} \text { in step } 7 \text { and } \mathbf{B}_{t}^{-1} \text { from Sec. III.A. }\end{array}$ \\
\hline 1: & $\gamma_{t \mid t-1} \leftarrow \gamma_{t-1 \mid t-1}$ from step 13 \\
2: & $\beta_{t \mid t-1} \leftarrow \beta_{t-1 \mid t-1}$ from step 13 \\
3: & $\delta_{t \mid t-1} \leftarrow \gamma_{t \mid t-1}, r_{t-1 \mid t-1}$ from steps 1,13 \\
4: & $\epsilon_{t \mid t-1} \leftarrow \beta_{t \mid t-1}, s_{t-1 \mid t-1}$ from steps 2,13 \\
5: & $r_{t \mid t-1} \leftarrow \delta_{t \mid t-1}, \epsilon_{t \mid t-1}$ from steps 3,4 \\
6: & $z_{t \mid t-1} \leftarrow r_{t \mid t-1}, n_{t \mid-}$ from step 5 and input (b) \\
7: & $s_{t \mid t}, z_{t \mid t} \leftarrow s_{t \mid t-1}, z_{t \mid t-1}, y_{t}$ from 6 and inputs (a), (c) \\
8: & $r_{t \mid t},\left[n_{t \mid t} \leftarrow \leftarrow r_{t \mid t-1}, n_{t \mid-}, z_{t \mid t}\right.$ from 5,7 and input (b) \\
9: & $\epsilon_{t \mid t}^{\prime} \leftarrow \beta_{t \mid t-1}, s_{t-1 \mid t}$ from step 2 and input (d) \\
10: & $\delta_{t \mid t}, \epsilon_{t \mid t} \leftarrow \delta_{t \mid t-1}, \epsilon_{t \mid t}^{\prime}, r_{t \mid t}$ from steps $3,8,9$ \\
11: & $\gamma_{t \mid t},\left[r_{t-1 \mid t}\right] \leftarrow \gamma_{t \mid t-1}, r_{t-1 \mid t-1}, \delta_{t \mid t}$ from steps $1,10,13$ \\
12: & $\beta_{t \mid t},\left[s_{t-1 \mid t} \leftarrow \leftarrow \beta_{t \mid t-1}, s_{t-1 \mid t}, \epsilon_{t \mid t}\right.$ from 2,10 and input (d) \\
13: & $r_{t-1 \mid t-1}, \gamma_{t-1 \mid t-1}, \beta_{t-1 \mid t-1} \leftarrow r_{t \mid t}, \gamma_{t \mid t}, \beta_{t \mid t}$ \\
\hline
\end{tabular}

For clarity, from this point onwards in Sec. III.C, the time subscript is included only if it differs from $t \mid t-1$. The prior and posterior time subscripts are presented in Table II.

The algorithm estimates the total distubance, $z_{t}$, from (3) in step 6 using similar equations to step 5. A two-dimensional Gaussian distribution is used for $p(r, n)$ and independence is assumed between $r$ and $n$. Hence, $p(r, n)=p(r) p(n)$. The phase-sensitive algorithm assumes that the phase difference, $\zeta$, between the two disturbance sources, $r$ and $n$, is uniformly distributed and independent of their magnitudes. Using a similar procedure to that used for $r$ in (23), we can transform $Z_{t}=$ $R_{t}+N_{t}$ from (3) into the form $e^{2 z}=e^{2 r}+e^{2 n}+2 \cos (\zeta) e^{r+n}$ to obtain $z=0.5 \log \left(e^{2 r}+e^{2 n}+2 \cos (\zeta) e^{r+n}\right)$. Hence,

$$
\begin{aligned}
\mathbb{E}\{z\} & =\int_{\zeta=0}^{\pi} \frac{1}{\pi} \int_{r, n} 0.5 \log \left(e^{2 r}+e^{2 n}+2 \cos (\zeta) e^{r+n}\right) \\
& \times p(r) p(n) d r d n d \zeta \\
\mathbb{E}\left\{z^{2}\right\} & =\int_{\zeta=0}^{\pi} \frac{1}{\pi} \int_{r, n}\left(0 . 5 \operatorname { l o g } \left(e^{2 r}+e^{2 n}\right.\right. \\
& \left.\left.+2 \cos (\zeta) e^{r+n}\right)\right)^{2} \times p(r) p(n) d r d n d \zeta
\end{aligned}
$$

where $K_{(r, n)}$ sigma points are utilised to evaluate the inner integral over $(r, n)$ [59] and $K_{\zeta}$ the outer integral over $\zeta$.

The algorithm performs noise suppression with steps 6 and 7. Step 7 decomposes $y_{t}$ into $s_{t}$ and $z_{t}$, as shown in Fig. 2. 
Step 7 performs the first signal decomposition, $y_{t}$ into $s_{t}$ and $z_{t}$, when propagating backwards through the signal model in Fig. 2. Step 7 applies the observation constraint, $y_{t}$, according to (3). As in [53] and [57], the variables are first transformed according to $(s, z, \lambda) \Rightarrow(u, y, \lambda)$ where $u=z-s$ and $\lambda=$ $\angle S-\angle Z$. This variable transformation is performed to allow the imposition of the real-valued scalar observation, $y_{t}$.

Using a similar procedure to that used for $z$ in (25), we can transform $Y_{t}=S_{t}+Z_{t}$ in (3) to obtain an expression for the noisy reverberant log-amplitude spectrum, $y$, as $y=$ $s+0.5 \log (1+\exp (2(z-s))+2 \cos (\lambda) \exp (z-s))$. The KF update step assumes that $\angle Z$ is uniformly distributed, $\angle Z \sim$ $U(-\pi, \pi)$. Hence, $\lambda \sim U(-\pi, \pi)$. The first two moments of the posteriors of $s_{t}$ and $z_{t}$ are computed in step $7 \mathrm{using}$

$$
\begin{aligned}
& \mathbb{E}\left\{s_{t}^{m_{1}} z_{t}^{m_{2}} \mid \mathcal{Y}_{t}\right\} \\
& =\int_{\lambda=-\pi}^{\pi} \int_{u=-\infty}^{\infty} s^{m_{1}} z^{m_{2}} p\left(u, \lambda \mid y_{t}\right) d u d \lambda \\
& \propto \frac{1}{|\Delta|} \int_{\lambda=-\pi}^{\pi} p(\lambda) \int_{u=-\infty}^{\infty} s^{m_{1}} z^{m_{2}} p(s, z) d u d \lambda \\
& \propto \int_{\lambda=0}^{\pi} \int_{u=-\infty}^{\infty} s^{m_{1}} z^{m_{2}} p(s) p(z) d u d \lambda
\end{aligned}
$$

where $s=-0.5 u+y-0.5 \log (2 \cosh (u)+2 \cos (\lambda))$ and $z=$ $s+u$ [52]. The Jacobian determinant is $\Delta=-1$. The moment indices, $m_{1}$ and $m_{2}$, are integers and $0 \leq m_{1}, m_{2} \leq 2$.

The first two moments of the posterior distributions of $s_{t}$ and $z_{t}$ are estimated. In (27), the priors of the speech and of the noise and late reverberation are assumed to be independent, i.e. $p(s, z)=p(s) p(z)$. In addition, in (27), $K_{\lambda}$ weighted sigma points are used to evaluate the outer integral over $\lambda$ [52].

Step 8 performs the second signal decomposition, $z_{t}$ into $r_{t}$ and $n_{t}$, when propagating backwards through the proposed signal model in Fig. 2. Step 8 decomposes $z_{t \mid t}$ into $r_{t}$ and $n_{t}$, according to (3), estimating both $r_{t \mid t}$ and $n_{t \mid t}$. It performs an integral over $z_{t}$ where the integrand is similar to step 7 and (27) and to the KF update step in [53] [57]. Instead of a scalar observation, as in step 7 , the observation in step 8 is a distribution; step 8 performs an outer integral over the observation distribution and the integrand is similar to step 7. Step 8 uses $\mathbb{E}\left\{r^{m}\right\}=\int_{z_{t}} \mathbb{E}\left\{r^{m} \mid z_{t}\right\} d z_{t}$ where $m \in \mathbb{N}_{0}$ and $0 \leq m \leq 2$. Step 7 computes a two-dimensional integral over the variables of $(z-s)$ and of the phase difference between $S$ and $Z$, using $y_{t}$ that reduces the probability space from three to two dimensions. In step 8, the KF algorithm calculates a threedimensional integral over: (a) $(r-n)$, (b) the phase difference, $\iota=\angle R-\angle N$, between $R$ and $N$, and (c) the posterior of $z_{t}$. Next, assuming $\angle N \sim U(-\pi, \pi)$ [52], step 8 computes

$$
\begin{aligned}
& \mathbb{E}\left\{r_{t}^{m_{1}} n_{t}^{m_{2}} \mid \mathcal{Y}_{t}\right\} \propto \int_{z=-\infty}^{\infty} p_{z_{t \mid t}}(z) \\
& \times \int_{\iota=0}^{\pi} \int_{u^{\prime}=-\infty}^{\infty} r^{m_{1}} n^{m_{2}} p(r) p(n) d u^{\prime} d \iota d z
\end{aligned}
$$

where $u^{\prime}=(r-n)$ and $\iota \sim U(-\pi, \pi)$. In (28), $z=r+$ $0.5 \log (1+\exp (2(n-r))+2 \cos (\iota) \exp (n-r)), r=-0.5 u^{\prime}+$ $z-0.5 \log \left(2 \cosh \left(u^{\prime}\right)+2 \cos (\iota)\right)$ and $n=r+u^{\prime}$ [52]. $K_{\iota}$ weighted sigma points are used to evaluate the integral over $\iota$ and $K_{z_{t \mid t}}$ sigma points to evaluate the integral over $z$ [59]. In step 8, the posterior of $n_{t}$ is estimated using (28) but is not used in the KF recursion. In Table II, the signals in square brackets are computed but not used in the KF recursion.

Step 9 determines a preliminary estimate of the posterior distribution of the new reverberation component, $\epsilon_{t}$, whose final estimate is calculated in step 10 . This preliminary estimate, denoted $\epsilon_{t \mid t}^{\prime}$, combines an updated estimate of the previous frame's speech, $s_{t-1 \mid t}$, with the prior estimate of the reverberation parameter, $\beta_{t \mid t-1}$. The sub-indexes $t \mid t-1$ and $t-1 \mid t$ give rise to the sub-index $t \mid t$. From (5), $\epsilon_{t}$ is the sum of two Gaussian random variables that are assumed to be independent and so we add their means and variances to obtain $\epsilon_{t \mid t}^{\prime}=\beta_{t \mid t-1}+s_{t-1 \mid t}$ and $\Sigma_{t \mid t}^{\left(\epsilon^{\prime}\right)}=\Sigma_{t \mid t-1}^{(\beta)}+\Sigma_{t-1 \mid t}^{(\mathrm{s})}$.

Step 10 decomposes the reverberation, $r_{t}$, into a new reverberation component, $\epsilon_{t}$ and an old decaying reverberation component, $\delta_{t}$, using (1), (4) and (5). Step 10 uses the prior distributions for these two components from steps 3 and 9. Step 10 performs the same operation as step 8. In analogy to step 7, the variable transformations in steps 8 and 10 are $(r, n, \iota) \Rightarrow\left(u^{\prime}, z, \iota\right)$ and $(\delta, \epsilon, \xi) \Rightarrow\left(u^{\prime \prime}, r, \xi\right)$, respectively. In step 10, the KF algorithm performs an integral over $r$ where the integrand is similar to the KF update step in [53]. Step 10 estimates the posterior distributions of $\delta$ and $\epsilon$; it estimates both $\delta_{t \mid t}$ and $\epsilon_{t \mid t}$ using $\delta_{t \mid t-1}$ and $\epsilon_{t \mid t}^{\prime}$. Step 10 computes

$$
\begin{aligned}
& \mathbb{E}\left\{\delta_{t}^{m_{1}} \epsilon_{t}^{m_{2}} \mid \mathcal{Y}_{t}\right\} \propto \int_{r=-\infty}^{\infty} p_{r_{t \mid t}}(r) \\
& \times \int_{\xi=0}^{\pi} \int_{u^{\prime \prime}=-\infty}^{\infty} \delta^{m_{1}} \epsilon^{m_{2}} p(\delta) p_{\epsilon_{t \mid t}^{\prime}}(\epsilon) d u^{\prime \prime} d \xi d r
\end{aligned}
$$

where $u^{\prime \prime}=(\delta-\epsilon)$ and $\xi \sim U(-\pi, \pi)$ is the phase difference of the STFT-domain signals of $\delta$ and $\epsilon$. In (29), $\delta=-0.5 u^{\prime \prime}+$ $r-0.5 \log \left(2 \cosh \left(u^{\prime \prime}\right)+2 \cos (\xi)\right)$ and $\epsilon=\delta+u^{\prime \prime}$ for the inverse transformation of $(\delta, \epsilon, \xi) \Rightarrow\left(u^{\prime \prime}, r, \xi\right)$ [52]. Also, $r=\delta+$ $0.5 \log (1+\exp (2(\epsilon-\delta))+2 \cos (\xi) \exp (\epsilon-\delta))$. We decompose $r_{t \mid t}$ into old reverberation and new reverberation, estimating both $\delta_{t \mid t}$ and $\epsilon_{t \mid t}$. $K_{\xi}$ sigma points are used to evaluate the integral over $\xi$ and $K_{r_{t \mid t}}$ sigma points the integral over $r$.

Steps 10-12 perform the final signal decomposition, $r_{t}$ into $\gamma_{t}$ and $\beta_{t}$, when propagating backwards through the signal model in Fig. 2. In steps 11 and 12, the KF algorithm computes the first two moments of the posterior distributions of $\gamma_{t}$ and $\beta_{t}$. In step 11, the algorithm performs an integral over $\delta_{t}$ using weighted sigma points where the integrand models the addition of two random variables in the log-amplitude spectral domain, $\gamma_{t}$ and $r_{t-1}$. Likewise, in step 12, the algorithm performs an integral over $\epsilon_{t}$ using sigma points where the integrand models the addition of two random variables in the log-amplitude spectral domain, $\beta_{t}$ and $s_{t-1}$. Steps 11 and 12 perform a linear $\mathrm{KF}$ update and impose a straight line observation constraint because the signal model is that $\gamma_{t}$ and $r_{t-1}$ are additive and that $\beta_{t}$ and $s_{t-1}$ are additive, according to (4) and (5).

In step 11, the non-linear KF algorithm decomposes the old reverberation component, $\delta_{t}$, according to (4) into the sum of a reverberation parameter, $\gamma_{t}$, and the previous frame's reverberation, $r_{t-1}$. We now define $\mathbf{x}_{1} \sim \mathrm{N}\left(\mathbf{x}_{1} ; \boldsymbol{\mu}_{1}, \mathbf{S}_{1}\right)$ where $\mathbf{x}_{1}=\left(\gamma_{t}, r_{t-1}, w_{t}\right)^{T} \in \mathbb{R}^{3}$, where $w_{t}$ is zero-mean Gaussian with variance $\Sigma_{t}^{(\delta)}, \boldsymbol{\mu}_{1}=\left(\gamma_{t \mid t-1}, r_{t-1 \mid t-1}, 0\right)^{T} \in \mathbb{R}^{3}$ and 
$\mathbf{S}_{1}=\operatorname{diag}\left(\left(\Sigma_{t \mid t-1}^{(\gamma)}, \Sigma_{t-1 \mid t-1}^{(\mathrm{r})}, \Sigma_{t \mid t}^{(\delta)}\right)^{T}\right) \in \mathbb{R}^{3 \times 3}$ where $\operatorname{diag}(\mathbf{j})$ is a diagonal matrix with the elements of the vector $\mathbf{j}$ on the main diagonal. If $\mathbf{x}_{1} \sim \mathrm{N}\left(\mathbf{x}_{1} ; \boldsymbol{\mu}_{1}, \mathbf{S}_{1}\right)$, then we can write,

$$
\begin{aligned}
\left(\mathbf{x}_{1} \mid \mathbf{g}^{T} \mathbf{x}_{1}=\delta_{t \mid t}\right) \sim & \mathrm{N}\left(\mathbf{x}_{1} ;\left(\mathbf{I}-\mathbf{H}_{1} \mathbf{g}^{T}\right) \boldsymbol{\mu}_{1}+\mathbf{H}_{1} \delta_{t \mid t},\right. \\
& \left.\left(\mathbf{I}-\mathbf{H}_{1} \mathbf{g}^{T}\right) \mathbf{S}_{1}\right)
\end{aligned}
$$

where $\mathbf{H}_{1}=\mathbf{S}_{1} \mathbf{g}\left(\mathbf{g}^{T} \mathbf{S}_{1} \mathbf{g}\right)^{-1}$ and $\mathbf{g}=(1,1,-1)^{T} \in \mathbb{R}^{3}[60]$. We compute $\left(\mathbf{I}-\mathbf{H}_{1} \mathbf{g}^{T}\right) \boldsymbol{\mu}_{1}+\mathbf{H}_{1} \delta_{t \mid t} \in \mathbb{R}^{3}$ and $\left(\mathbf{I}-\mathbf{H}_{1} \mathbf{g}^{T}\right) \mathbf{S}_{1} \in$ $\mathbb{R}^{3 \times 3}$ and set $\gamma_{t \mid t}$ equal to the first element of this computed mean and $\Sigma_{t \mid t}^{(\gamma)}$ equal to the first element of this variance.

Likewise, for step 12, we define $\mathbf{x}_{2} \sim \mathrm{N}\left(\mathbf{x}_{2} ; \boldsymbol{\mu}_{2}, \mathbf{S}_{2}\right)$ where $\mathbf{x}_{2}=\left(\beta_{t}, s_{t-1}, q_{t}\right)^{T} \in \mathbb{R}^{3}, q_{t}$ is zero-mean Gaussian with variance $\Sigma_{t}^{(\epsilon)}, \boldsymbol{\mu}_{2}=\left(\beta_{t \mid t-1}, s_{t-1 \mid t}, 0\right)^{T} \in \mathbb{R}^{3}$ and, using (5), $\mathbf{S}_{2}=\operatorname{diag}\left(\left(\Sigma_{t \mid t-1}^{(\beta)}, \Sigma_{t-1 \mid t}^{(\mathrm{s})}, \Sigma_{t \mid t}^{(\epsilon)}\right)^{T}\right) \in \mathbb{R}^{3 \times 3}$. Next, we use

$$
\begin{aligned}
\left(\mathbf{x}_{2} \mid \mathbf{g}^{T} \mathbf{x}_{2}=\epsilon_{t \mid t}\right) \sim & \mathrm{N}\left(\mathbf{x}_{2} ;\left(\mathbf{I}-\mathbf{H}_{2} \mathbf{g}^{T}\right) \boldsymbol{\mu}_{2}+\mathbf{H}_{2} \epsilon_{t \mid t},\right. \\
& \left.\left(\mathbf{I}-\mathbf{H}_{2} \mathbf{g}^{T}\right) \mathbf{S}_{2}\right)
\end{aligned}
$$

where $\mathbf{H}_{2}=\mathbf{S}_{2} \mathbf{g}\left(\mathbf{g}^{T} \mathbf{S}_{2} \mathbf{g}\right)^{-1}$ [60]. The KF algorithm computes $\left(\mathbf{I}-\mathbf{H}_{2} \mathbf{g}^{T}\right) \boldsymbol{\mu}_{2}+\mathbf{H}_{2} \epsilon_{t \mid t} \in \mathbb{R}^{3}$ and $\left(\mathbf{I}-\mathbf{H}_{2} \mathbf{g}^{T}\right) \mathbf{S}_{2} \in \mathbb{R}^{3 \times 3}$ and sets $\beta_{t \mid t}$ equal to the first element of this computed mean and $\Sigma_{t \mid t}^{(\beta)}$ equal to the first element of this computed variance. In steps 11 and 12 , we compute $\gamma_{t \mid t}, \Sigma_{t \mid t}^{(\gamma)}$ and $\beta_{t \mid t}, \Sigma_{t \mid t}^{(\beta)}$, respectively, using both $p\left(\gamma_{t}, r_{t-1} \mid \delta_{t \mid t}+w_{t}\right)$ and $p\left(\beta_{t}, s_{t-1} \mid \epsilon_{t \mid t}+q_{t}\right)$.

Finally, step 13 in Table II applies a one-frame delay, $z^{-1}$, in order to continue the KF recursion, as shown in Fig. 3.

\section{Discussion of the Non-Linear KF Update Step}

Section III.C presented the operations performed in the update step. This section discusses these operations that track the log-spectra of speech and reverberation blindly and adaptively. Steps 7 to 12 of Table II use $y_{t}$ to update in sequence the probability distributions of $s_{t}, z_{t}, r_{t}, n_{t}, \delta_{t}, \epsilon_{t}, \gamma_{t}$ and $\beta_{t}$ by tracing backwards through the block diagram in Fig. 2 .

According to the 13 steps, $\gamma_{t}$ and $\beta_{t}$ are affected by $y_{t}$ through a series of operations that estimate the first two moments of the posterior distributions. The estimate of $\gamma_{t}$ is affected by the previous estimate of $\beta_{t}$ because of the sequence of operations $\beta_{t-1 \mid t-1} \Rightarrow \beta_{t \mid t-1} \Rightarrow \epsilon_{t \mid t}^{\prime} \Rightarrow \delta_{t \mid t} \Rightarrow \gamma_{t \mid t}$. Likewise, the estimate of $\beta_{t}$ depends on the previous estimate of $\gamma_{t}$ because $\gamma_{t-1 \mid t-1} \Rightarrow \gamma_{t \mid t-1} \Rightarrow \delta_{t \mid t-1} \Rightarrow \epsilon_{t \mid t} \Rightarrow \beta_{t \mid t}$.

Step 7 starts from a distribution in the three-dimensional probability space, performs a specific variable transformation, imposes the KF observation constraint [52] and ends with a distribution in the two-dimensional probability space. Step 7 computes both $s_{t \mid t}$ and $z_{t \mid t}$ and is based on the non-linear KF update step presented in [53]. The algorithm presented in this section, for joint denoising and dereverberation, generalizes the algorithm presented in [53]. It can be stated that the update step described in [52] could be used in step 7 in Table II in lieu of the update step detailed in [53]. This would result in tracking the speech phase and performing dereverberation.

In steps 7 and 8 , the algorithm estimates $s_{t \mid t}, r_{t \mid t}$ and $n_{t \mid t}$ and their variances, $\Sigma_{t \mid t}^{(s)}, \Sigma_{t \mid t}^{(r)}$ and $\Sigma_{t \mid t}^{(n)}$. The posterior means of speech, reverberation and noise given $y_{t}$ are more accurate estimates of speech, reverberation and noise than the estimates produced by the unshaded peripheral blocks in Fig. 3.
The proposed 13 steps do not include the speech prediction step shown in Fig. 3, which is used to calculate $s_{t-1 \mid t}$ that is needed in steps 9 and 12. After step 7, according to Fig. 3 and Sec. III.B, recorrelation of the speech KF state is performed. Using $s_{t \mid t}$, from the recorrelation step, $s_{t-1 \mid t}$ is obtained and $s_{t-1 \mid t}$ is used as a better estimate of $s_{t-1}$ than $s_{t-1 \mid t-1}$.

Steps 11 and 12 estimate $\gamma_{t \mid t}$ and $\beta_{t \mid t}$ together with their variances, $\Sigma_{t \mid t}^{(\gamma)}$ and $\Sigma_{t \mid t}^{(\beta)}$. Joint denoising and dereverberation using external reverberation parameter estimation could be performed using a simplified version of the algorithm in Fig. 3. An external $T_{60}$ and DRR estimator, such as [61] [62], could be used instead of tracking $\gamma_{t}$ and $\beta_{t}$ in (4) and (5), outside the $\mathrm{KF}$ loop, not in an online adaptive manner. Batch processing could be used for reverberation parameter estimation and the $T_{60}$ and the DRR could be assumed time-invariant [15].

\section{E. The Priors for the Reverberation Parameters}

This section describes the priors for $\gamma_{t}$ and $\beta_{t}$ based on [63] [64] and [61]. These priors are imposed using GaussianGaussian multiplication; $\gamma_{t}$ is modelled with a Gaussian distribution and its internal prior is also a Gaussian. Likewise, $\beta_{t}$ is modelled with a Gaussian and its prior is a Gaussian.

The priors for $\gamma_{t}$ and $\beta_{t}$ are estimated from spectral logamplitude observations in the free decay region (FDR), which comprises $M_{t}$ consecutive frames with decreasing energy. We define the look-ahead time interval, $C$, and the frame index $l=t-M_{t}+C+1, t-M_{t}+C+2, \ldots, t+C$. The least squares (LS) fit to the FDR is found using $r_{l}=\theta_{1} x_{l}+\theta_{2}$ where $x_{l}$ is the time index in seconds and depends on $L$. The parameters of the straight line are the slope, $\theta_{1}$, and the y-intercept, $\theta_{2}$. For clarity, the time subscript, $t$, is omitted from $\theta_{1}$ and $\theta_{2}$. We define $\boldsymbol{\theta}=\left(\theta_{1} \theta_{2}\right)^{T} \in \mathbb{R}^{2}$. Its Gaussian distribution is

$$
\begin{aligned}
& N\left(\boldsymbol{\theta} ; \boldsymbol{\mu}_{\theta}, \boldsymbol{\Sigma}_{\theta}\right) \propto\left|\boldsymbol{\Sigma}_{\theta}\right|^{-0.5} \\
& \times \exp \left(-0.5\left(\boldsymbol{\theta}-\boldsymbol{\mu}_{\theta}\right)^{T} \boldsymbol{\Sigma}_{\theta}^{-1}\left(\boldsymbol{\theta}-\boldsymbol{\mu}_{\theta}\right)\right)
\end{aligned}
$$

where $\boldsymbol{\mu}_{\theta} \in \mathbb{R}^{2}$ and the covariance matrix is $\boldsymbol{\Sigma}_{\theta} \in \mathbb{R}^{2 \times 2}$

The $\log$-likelihood of $\boldsymbol{\theta}$ is given by $l(\boldsymbol{\theta})=\mathrm{c}_{1}-0.5(\boldsymbol{\theta}$ $\left.\boldsymbol{\mu}_{\theta}\right)^{T} \boldsymbol{\Sigma}_{\theta}^{-1}\left(\boldsymbol{\theta}-\boldsymbol{\mu}_{\theta}\right)=\mathrm{c}_{2}-0.5 \sum_{\tau=t-M_{t}+C+1}^{t+C}\left(\left(\theta_{1} x_{\tau}+\theta_{2}-\right.\right.$ $\left.\left.r_{\tau}\right)^{T}\left(\Sigma_{\tau}^{(r)}\right)^{-1}\left(\theta_{1} x_{\tau}+\theta_{2}-r_{\tau}\right)\right)$ where $\mathrm{c}_{1}$ and $\mathrm{c}_{2}$ are constants.

We define the vectors $\mathbf{x} \in \mathbb{R}^{M_{t}}$ and $\mathbf{r} \in \mathbb{R}^{M_{t}}$ from $x_{l}$ and $r_{l}$, respectively. We define $\boldsymbol{\Sigma}_{r} \in \mathbb{R}^{M_{t} \times M_{t}}$ as the covariance matrix of $\mathbf{r}$. The regression coefficients as a Gaussian distribution are $N\left(\boldsymbol{\theta} ; \boldsymbol{\mu}_{\theta}, \boldsymbol{\Sigma}_{\theta}\right)$ where $\boldsymbol{\mu}_{\theta}=\mathbf{A}^{-1} \mathbf{b}$ and $\boldsymbol{\Sigma}_{\theta}=\mathbf{A}^{-1}$,

$$
\begin{aligned}
\mathbf{A} & =\left(\begin{array}{cc}
\mathbf{x}^{T} \boldsymbol{\Sigma}_{r}^{-1} \mathbf{x} & \mathbf{x}^{T} \boldsymbol{\Sigma}_{r}^{-1} \mathbf{1} \\
\mathbf{1}^{T} \boldsymbol{\Sigma}_{r}^{-1} \mathbf{x} & \mathbf{1}^{T} \boldsymbol{\Sigma}_{r}^{-1} \mathbf{1}
\end{array}\right), \\
\mathbf{b} & =\left(\begin{array}{c}
\mathbf{x}^{T} \boldsymbol{\Sigma}_{r}^{-1} \mathbf{r} \\
\mathbf{1}^{T} \boldsymbol{\Sigma}_{r}^{-1} \mathbf{r}
\end{array}\right)
\end{aligned}
$$

where $\mathbf{1} \in \mathbb{R}^{M_{t}}$ is a vector of ones. Figure 4 shows the $\log$ likelihood of the Gaussian distribution of $\boldsymbol{\theta}$ with mean $\boldsymbol{\mu}_{\theta}$ and variance $\boldsymbol{\Sigma}_{\theta}$ when the correlation between $\theta_{1}$ and $\theta_{2}$ is strong. We note that the maximum likelihood solution for the mean of $\theta_{1}$, when $\theta_{2}$ is not considered, can be found in [65].

The noisy reverberation, $z_{t}$, is the observation and, in this case, our aim is to find $r_{t}$ and $\Sigma_{t}^{(r)}$. We denote the noise floor in the power spectral domain by $|N|^{2}$ and we define the RNR 
as $\mathrm{RNR}=\exp (2(r-n))$. In a FDR, at high RNRs and when $|N|^{2}$ is low, $r_{t} \approx z_{t}$ because $z=r+0.5 \log \left(1+\mathrm{RNR}^{-1}+\right.$ $2 \cos (\zeta) \mathrm{RNR}^{-0.5}$ ) from (25) and (26), using $\zeta$ as in [52].

To find $r_{t}$ and $\Sigma_{t}^{(r)}$ given the observed $z_{t}$, we first employ a minimum MSE (MMSE) algorithm, such as the Log-MMSE estimator [37], to attenuate the noise and estimate the signal's spectrum, as in [61], and then perform a KF update step and use $z_{t}$ as the KF observation. For the linear KF [66] [67], when the observation noise covariance matrix tends to zero at high RNRs, then the mean of the state goes to the KF observation and the variance of the state goes to zero. A solution to the problem of finding $r_{t}$ and $\Sigma_{t}^{(r)}$ is to set $r_{t}=z_{t}$ and to also set $\Sigma_{t}^{(r)}$ equal to a fixed small value, such as $1 \mathrm{~dB}^{2}$.

The $T_{60}$ can be estimated from $\theta_{1}$ using $T_{60}=-\frac{3 \log (10)}{\theta_{1}}$, where $\theta_{1}$ is computed from FDR data in the log-magnitude spectral domain [64]. The KF algorithm, which models $\gamma_{t}$ with a Gaussian distribution, uses a Gaussian prior for $\gamma$ with mean $L \mu_{\theta_{1}}$ and variance $L^{2} \Sigma_{\theta_{1}}$ because $\gamma=L \theta_{1}$. Using $\gamma=L \theta_{1}$, $\theta_{1}$ is directly mapped to $\gamma_{t}$, without going via the $T_{60}$.

The algorithm estimates a prior for $\beta_{t}$ at the same time as the prior for $\gamma_{t}$. Figure 5 depicts $y_{t}, s_{t}$ and $r_{t}$ over time, at $1 \mathrm{kHz}$, when the noise is white, the reverberant speech to noise ratio (RSNR) is $20 \mathrm{~dB}$, the $T_{60}$ is $0.68 \mathrm{~s}$ and the DRR is $-2.52 \mathrm{~dB}$. In Fig. 5, $s_{t}$ and $r_{t}$ are the true speech and reverberation powers; the latter is computed by convolving the RIR without its first $30 \mathrm{~ms}$ with the speech signal [2]. If we choose an appropriate FDR, then (1) and (2) are related to the FDR and the first frame of the FDR to the DRR. Here, $\beta$ is computed from the difference between the log-amplitude of the first frame and the $r$-intercept of the LS fit, i.e. $\theta_{2}$. For the subtraction of two independent random variables, their means subtract and their variances add; $\theta_{2}$ has a mean and a variance and the log-amplitude of the first frame of the FDR has a mean and a fixed small variance. The prior for $\beta_{t}$ is given by

$$
\beta_{t}=r_{t-M_{t}+C+1}-\theta_{2} \text {. }
$$

We denote the mean of the internally computed Gaussian $\gamma_{t}$ prior by $\gamma_{t \mid t-1}^{\prime \prime}$ and its variance by $\Sigma_{t \mid t-1}^{\left(\gamma^{\prime \prime}\right)}$. The KF uses

$$
\begin{aligned}
\gamma_{t \mid t-1} & =\frac{\gamma_{t \mid t-1}^{\prime} \Sigma_{t \mid t-1}^{\left(\gamma^{\prime \prime}\right)}+\gamma_{t \mid t-1}^{\prime \prime} \Sigma_{t \mid t-1}^{\left(\gamma^{\prime}\right)}}{\Sigma_{t \mid t-1}^{\left(\gamma^{\prime}\right)}+\Sigma_{t \mid t-1}^{\left(\gamma^{\prime \prime}\right)}}, \\
\Sigma_{t \mid t-1}^{(\gamma)} & =\frac{\Sigma_{t \mid t-1}^{\left(\gamma^{\prime}\right)} \Sigma_{t \mid t-1}^{\left(\gamma^{\prime \prime}\right)}}{\Sigma_{t \mid t-1}^{\left(\gamma^{\prime}\right)}+\Sigma_{t \mid t-1}^{\left(\gamma^{\prime \prime}\right)}} .
\end{aligned}
$$

Likewise, we denote the mean of the Gaussian $\beta_{t}$ prior by $\beta_{t \mid t-1}^{\prime \prime}$ and its variance by $\Sigma_{t \mid t-1}^{\left(\beta^{\prime \prime}\right)}$. For $\beta_{t}$ in (5) and Fig. 1,

$$
\begin{aligned}
\beta_{t \mid t-1} & =\frac{\beta_{t \mid t-1}^{\prime} \Sigma_{t \mid t-1}^{\left(\beta^{\prime \prime}\right)}+\beta_{t \mid t-1}^{\prime \prime} \Sigma_{t \mid t-1}^{\left(\beta^{\prime}\right)}}{\Sigma_{t \mid t-1}^{\left(\beta^{\prime}\right)}+\Sigma_{t \mid t-1}^{\left(\beta^{\prime \prime}\right)}}, \\
\Sigma_{t \mid t-1}^{(\beta)} & =\frac{\Sigma_{t \mid t-1}^{\left(\beta^{\prime}\right)} \Sigma_{t \mid t-1}^{\left(\beta^{\prime \prime}\right)}}{\Sigma_{t \mid t-1}^{\left(\beta^{\prime}\right)}+\Sigma_{t \mid t-1}^{\left(\beta^{\prime \prime}\right)}} .
\end{aligned}
$$

Blind $T_{60}$ estimation, when the RIR is unknown [68], is not straightforward. The KF algorithm estimates the $T_{60}$ using (32)-(39) and the decay rate of the LS fit to the FDR [64] so that the KF does not diverge and does not treat $r_{t}$ as $s_{t}$.

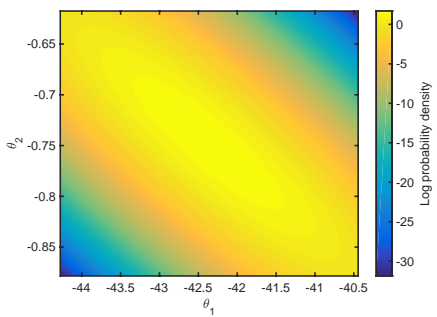

Figure 4. Plot of the log-likelihood of the Gaussian distribution of $\boldsymbol{\theta}$ in (32). $\boldsymbol{\Sigma}_{\theta}=\mathbf{A}^{-1}$ is non-diagonal. $\mathbf{A}$ is computed in (33) from the FDR.

\section{F. The Peripheral Blocks of the Algorithm}

This section describes the unshaded blocks in Fig. 3 that lie outside the KF loop. The algorithm uses pre-cleaning before performing speech AR modelling, as in [52] and [67], and pre-cleaning is also used in [50] [51] and in [10] [54]. The "Speech pre-cleaning" block in Fig. 3 affects the "Speech KF prediction" block and not the observation of the "KF Update" block, i.e. $y_{t}$, which is used in step 7 in Table II. Speech amplitude spectrum pre-cleaning, which is scale independent [11], is performed before the "sliding-window" AR modeling in the log-spectral domain. The "pre-cleaning" block applies a conventional enhancement algorithm, such as the Log-MMSE enhancer [37] followed by the WPE dereverberation algorithm [69] [70], to the noisy speech so that speech AR modeling can be performed. The speech AR coefficients are estimated from noisy reverberant speech and their estimate is biased [29] and this model mis-specification is fixed by pre-cleaning [50]. Although it would be possible to use the enhanced signal from the KF output when estimating the AR coefficients, we have chosen not to do so to avoid the risk of instability [71].

For the "Noise power estimation" block, an external noise power estimator, such as [72], is used. External noise estimation and log-normal noise power modelling, as in [73] [74], are used for $n_{t \mid-}$, which is then used in step 6 of Table II. The purpose of the external noise estimator is to remove ambiguity when jointly estimating the log-magnitude spectra of speech, noise and reverberation in steps 7 and 8 . The noise estimator, which is out of the KF loop in Fig. 3, provides a mechanism that helps the algorithm distinguish between speech, noise and reverberation. Without additional information, estimating the first two moments of the posterior distributions of $s_{t}, n_{t}$ and $r_{t}$ given the real-valued $y_{t}$ is an ill-conditioned problem.

In summary, the KF algorithm tracks speech and reverberation in the log-magnitude spectral domain along with $\gamma_{t}$ and $\beta_{t}$, as described in Fig. 3 and in the signal model in Fig. 2.

\section{IMPLEMENTATION, TESTING AND VALIDATION}

We use acoustic frames of length $32 \mathrm{~ms}$ and an acoustic frame increment of $L=8 \mathrm{~ms}$ in (2). We also use modulation frames of $64 \mathrm{~ms}$ and a modulation frame increment of $8 \mathrm{~ms}$ [52] [54]. The dimension of the speech $\mathrm{KF}$ state, $\mathbf{s}_{t}$, in (6) is $p=2$ resulting in a total KF state dimension of 5 when the reverberation parameters, $r_{t}, \gamma_{t}$ and $\beta_{t}$, are included. In Fig. 3, for pre-cleaning, we use the Log-MMSE enhancer [37] 


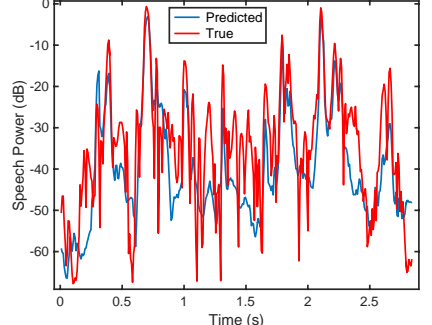

(a)

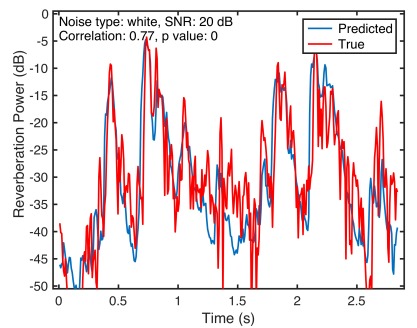

(c)

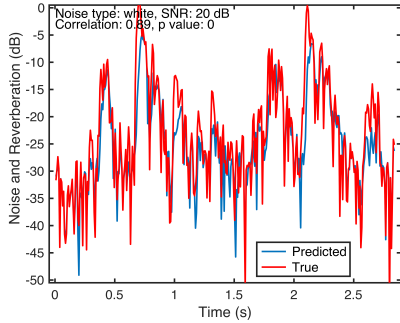

(b)
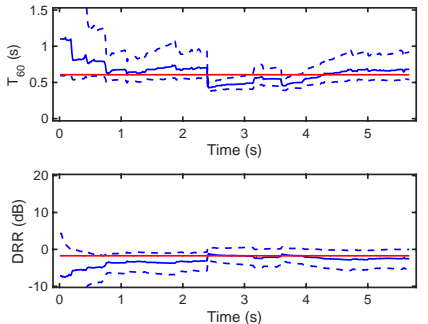

(d)
Figure 6. Plot of the predicted and true: (a) speech power, i.e. $s_{t \mid t}$ and the true $s_{t}$, at $1 \mathrm{kHz}$, (b) noise and reverberation power, i.e. $z_{t \mid t}$ and the true $z_{t}$ at $1 \mathrm{kHz}$, (c) reverberation power, i.e. $r_{t \mid t}$ and the true $r_{t}$, at $1 \mathrm{kHz}$, and (d) $T_{60}$ and DRR reverberation parameters. In (a)-(d), the $T_{60}$ is $0.61 \mathrm{~s}$ and the DRR is $-1.74 \mathrm{~dB}$, while the noise type is white and the RSNR is $20 \mathrm{~dB}$

(implemented in [75]) followed by the WPE dereverberation algorithm [69] [70]. In the "Noise power estimation" block of Fig. 3, we use the unbiased MMSE-based noise estimator from [72] (implemented in [75]), as in [10], [11] and [52].

In Sec. III.B, the random-walk parameters, $Q_{\gamma}$ and $Q_{\beta}$, are set to fixed values that are chosen so that the standard deviation corresponds to a rate of change of the $T_{60}$ and the DRR of $10 \%$ per second. If it is known that the acoustic environment is static, the reverberation parameters can be taken to be timeinvariant and estimated offline. In this case, we can set $Q_{\gamma}=$ $Q_{\beta}=0$ and omit steps 11 and 12 from Table II. In realistic situations, however, the movement of people and the opening of doors or windows mean that the reverberation parameters are inconstant and should be tracked in the algorithm.

The outer integrals in Secs. III.B and III.C are performed using sigma points, as in [52]. The numbers of sigma points used in (23)-(29) are $K_{(\delta, \epsilon)}=K_{(r, n)}=K_{z_{t \mid t}}=K_{r_{t \mid t}}=3$ and $K_{\eta}=K_{\zeta}=K_{\lambda}=K_{\iota}=K_{\xi}=6$. For the latter cases, for $\xi$, the sigma points are at $\xi=\left(\left(1: K_{\xi}\right)-0.5\right) \frac{\pi}{K_{\xi}}$ and the weights are all equal to $\frac{1}{K_{\xi}}$ [52] [53]. With this choice of sigma points for $\xi$, the integral will be exact for an integrand that is a sum of terms of the form $\cos (n \xi)$ for $0 \leq n \leq 2 K_{\xi}+1$.

In Sec. III.E, for the $\gamma_{t}$ and $\beta_{t}$ priors, the look-ahead factor is $C=3$ frames. For the FDR that is comprised of frames with decreasing energy, $M_{t}$ is computed in every frame.

Figures 6(a)-(c) show the time variation of $s_{t}, z_{t}$ and $r_{t}$ in the $1 \mathrm{kHz}$ bin, as in [67]. Figure 6(a) depicts the predicted and true speech power, i.e. $s_{t \mid t}$ and the true $s_{t}$, and Fig. 6(b) the predicted and true noise plus reverberation power, i.e. $z_{t \mid t}$ and the true $z_{t}$. The correlation coefficient between the true $z_{t}$ and $z_{t \mid t}$ is 0.89 . Figure 6(c) shows the predicted and true reverberation power, i.e. $r_{t \mid t}$ and the true $r_{t}$. The correlation coefficient between the true $r_{t}$ and $r_{t \mid t}$ is $0.77(<0.89)$. The ordering of the graphs in Figs. 6(a)-(c) matches the ordering of the signal decompositions in the KF algorithm, in Table II. The noisy reverberant log-spectrum is first decomposed into $s_{t}$ and $z_{t}$ with step 7; Figs. 6(a) and (b) illustrate $s_{t \mid t}$ and $z_{t \mid t}$, respectively, over time. Then, $z_{t \mid t}$ is decomposed into $r_{t}$ and $n_{t}$ with step 8 and Fig. 6(c) depicts $r_{t \mid t}$ over time. Here, RSNR = $20 \mathrm{~dB}$ white noise, $T_{60}=0.61 \mathrm{~s}$ and DRR $=-1.74 \mathrm{~dB}$.

The $T_{60}$ and DRR estimates should converge to their true constant values when both the talker and the microphone are not moving and the frequency variations in the reflection coefficients are not modelled [76] [77]. The internally estimated priors for $\gamma_{t}$ and $\beta_{t}$ make the reverberation parameters converge over time. Figure $6(\mathrm{~d})$ shows the predicted and true $T_{60}$ and DRR reverberation parameters and the ground truth values are shown as horizontal lines [5]. The dashed lines are the signals' standard deviations computed from $\gamma_{t}$ and $\beta_{t}$.

\section{Evaluation of the Algorithm}

The KF algorithm is evaluated in terms of the perceptual evaluation of speech quality (PESQ) [78], the cepstral distance (CD) spectral divergence metric [79], the reverberation decay tail (RDT) dereverberation metric [80] and the STOI speech intelligibility metric [81] [82]. The ideal values of CD and RDT, which were also used in [20] [21], are zero. For evaluation, the TIMIT database [83], sampled at $16 \mathrm{kHz}$, and the RSG-10 noise database [84] are used. From the TIMIT database, 52 utterances are chosen. We use artificially-created reverberation with the image method [76] using the implementation in [85] [9]. The wall reflection coefficient is adjusted to vary the $T_{60}$ and the DRR. The adaptive KF algorithm is evaluated with noisy reverberant speech at various RSNRs [86], using random noise segments and white, babble and factory noises.

The KF algorithm is compared to the SPENDRED algorithm [20] [21], which jointly performs blind denoising and dereverberation, and to algorithms that sequentially perform denoising and dereverberation, specifically to the Log-MMSE estimator [37] followed by the WPE algorithm [70] [31] and to the Log-MMSE estimator followed by an inverse-filter (IF) dereverberation method based on $T_{60}$ and DRR estimates. For the Log-MMSE enhancer followed by the WPE algorithm, we also evaluated applying WPE followed by Log-MMSE but found that this reverse order leads to marginally worse enhancement results, averaging -0.02 in PESQ. For the IF, we blindly estimate the $T_{60}$ and the DRR for the entire utterance using the algorithm in [61] [62], whose implementation was generously provided by the author. As found in [1], the $T_{60}$ estimator in [61] had the best performance of the examined estimators. We apply a dereverberation post-filter to the output of a Log-MMSE enhancer using the $T_{60}$ and DRR estimates. Assuming that $a$ and $b$ in (2) are time-invariant and frequencyindependent, using a reverberation model in the power spectral domain [20] [21], if $\left|\hat{S}_{t}\right|^{2}$ is the output power from LogMMSE, then $\left|S_{t}\right|^{2}=(a-b)\left|S_{t-1}\right|^{2}+\left|\hat{S}_{t}\right|^{2}-a\left|\hat{S}_{t-1}\right|^{2}$.

For evaluation, the examined acoustic conditions are shown in Table III. Two different rooms with dimensions $5 \times 4 \times$ $4 \mathrm{~m}$ and $10 \times 7 \times 3 \mathrm{~m}$ are used. The distance between the microphone and the talker is $1.5 \mathrm{~m}$. Table III is sorted with 
Table III

List of the environments sorted with respect to the $T_{60}$ from A to I and from $\mathrm{J}$ to $\mathrm{V}$. The room dimensions are $5 \times 4 \times 4$ or $10 \times 7 \times 3 \mathrm{~m}$. The source-microphone distance is $1.5 \mathrm{~m}$. The wall reflection coefficient is adjusted to vary the $T_{60}$ and the DRR. For $a$ and $b$ in (2), $L=8 \mathrm{~ms}$.

\begin{tabular}{|c|c|c|c|c|c|}
\hline Index & $T_{60}(\mathbf{s})$ & DRR (dB) & $\operatorname{Room}(\mathbf{m} \times \mathbf{m} \times \mathbf{m})$ & $a$ & $b$ \\
\hline $\mathrm{A}$ & 0.18 & 8.43 & $5 \times 4 \times 4$ & 0.54 & 0.07 \\
\hline $\mathrm{B}$ & 0.25 & 5.78 & $5 \times 4 \times 4$ & 0.64 & 0.10 \\
\hline $\mathrm{C}$ & 0.33 & 3.13 & $5 \times 4 \times 4$ & 0.72 & 0.14 \\
\hline $\mathrm{D}$ & 0.40 & 1.69 & $5 \times 4 \times 4$ & 0.76 & 0.16 \\
\hline $\mathrm{E}$ & 0.47 & 0.25 & $5 \times 4 \times 4$ & 0.79 & 0.20 \\
\hline $\mathrm{F}$ & 0.54 & -0.74 & $5 \times 4 \times 4$ & 0.81 & 0.23 \\
\hline $\bar{G}$ & 0.61 & -1.74 & $5 \times 4 \times 4$ & 0.83 & 0.25 \\
\hline $\mathrm{H}$ & 0.64 & -2.13 & $5 \times 4 \times 4$ & 0.84 & 0.26 \\
\hline I & 0.68 & -2.52 & $5 \times 4 \times 4$ & 0.85 & 0.27 \\
\hline $\mathrm{J}$ & 0.21 & 8.07 & $10 \times 7 \times 3$ & 0.59 & 0.06 \\
\hline $\mathrm{K}$ & 0.31 & 2.74 & $10 \times 7 \times 3$ & 0.70 & 0.16 \\
\hline $\mathrm{L}$ & 0.40 & 0.17 & $10 \times 7 \times 3$ & 0.76 & 0.23 \\
\hline $\mathrm{M}$ & 0.50 & 0.11 & $10 \times 7 \times 3$ & 0.80 & 0.19 \\
\hline $\mathrm{N}$ & 0.59 & -0.73 & $10 \times 7 \times 3$ & 0.83 & 0.20 \\
\hline $\mathrm{O}$ & 0.64 & -0.95 & $10 \times 7 \times 3$ & 0.84 & 0.20 \\
\hline $\mathrm{P}$ & 0.69 & -1.12 & $10 \times 7 \times 3$ & 0.85 & 0.19 \\
\hline $\mathrm{Q}$ & 0.71 & -1.68 & $10 \times 7 \times 3$ & 0.86 & 0.21 \\
\hline $\mathrm{R}$ & 0.73 & -2.01 & $10 \times 7 \times 3$ & 0.86 & 0.22 \\
\hline $\mathrm{S}$ & 0.85 & -2.09 & $10 \times 7 \times 3$ & 0.88 & 0.19 \\
\hline $\mathrm{T}$ & 0.97 & -2.95 & $10 \times 7 \times 3$ & 0.89 & 0.22 \\
\hline $\bar{U}$ & 1.01 & -3.11 & $10 \times 7 \times 3$ & 0.90 & 0.20 \\
\hline $\mathrm{V}$ & 1.05 & -3.33 & $10 \times 7 \times 3$ & 0.90 & 0.22 \\
\hline
\end{tabular}

respect to the $T_{60}$ and the DRR from A to $\mathrm{I}$ and from $\mathrm{J}$ to $\mathrm{V}$. We plot the improvement in PESQ, $\triangle \mathrm{PESQ}$, against the index of the acoustic environment to evaluate the KF algorithm. The top axis shows the raw PESQ of the noisy reverberant speech and is monotonic from A to I and from J to V. Similar graphs are also plotted for the other metrics. The ordering of the legends matches that of the algorithms at low $T_{60}$ values.

Table III also shows the $a$ and $b$ reverberation values of the RIRs and we note that the SPENDRED algorithm [20] [21] assumes that $b \leq 1$. This is valid in Table III since $b<<1$.

\section{A. Overall Performance Against the $T_{60}$ and the DRR}

This section investigates the performance of the algorithm against the $T_{60}$ and the DRR. Figure 7 shows: (a) the $\triangle \mathrm{PESQ}$, where higher scores signify better speech quality, (b) the $\triangle \mathrm{CD}$, where lower values signify better speech quality, (c) the $\triangle \mathrm{RDT}$, where lower values signify better dereverberation, and (d) the $\Delta$ STOI, where higher scores signify better intelligibility. Figure 7 first presents the results that are related to speech quality, in (a) and (b). Then, it shows the dereverberation results, in (c), and the speech intelligibility results, in (d). The graphs are against the index of the acoustic environment and, hence, against the $T_{60}$ and the DRR. The average over the white, babble and factory noises at $20 \mathrm{~dB}$ RSNR is shown.

In Fig. 7(a), the top axis presents the raw PESQ of the unenhanced signal and is monotonic from $\mathrm{A}$ to $\mathrm{I}$ and from $\mathrm{J}$ to $\mathrm{V}$; there is no transition between index I and index $\mathrm{J}$.

From Fig. 7(a), the algorithm consistently yields improved PESQ quality in challenging conditions compared to the baselines. Compared to the unprocessed signal, the algorithm shows improved performance for all the examined $T_{60}$ range from 0.18 to $1.05 \mathrm{~s}$ and DRR range from 8.43 to $-3.33 \mathrm{~dB}$. Compared to the unprocessed signal, for a $T_{60}$ of 0.3 and $1 \mathrm{~s}$,

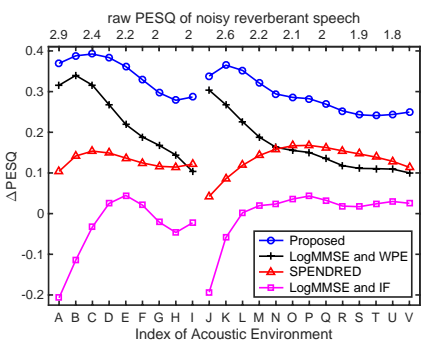

(a)

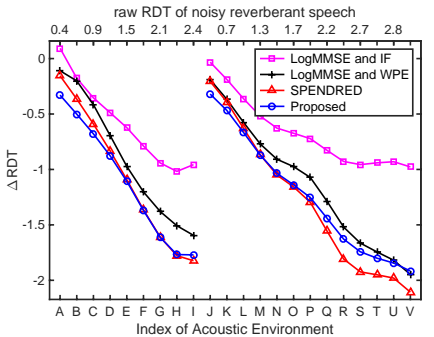

(c)

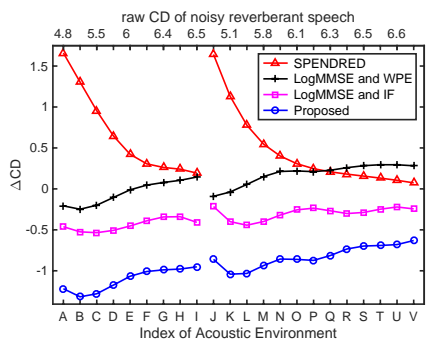

(b)

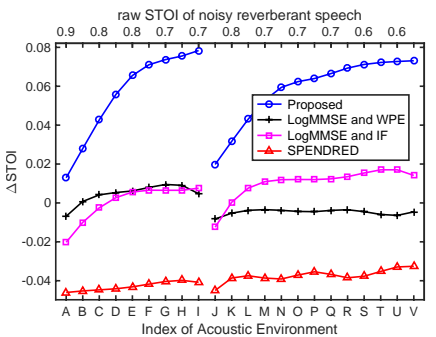

(d)
Figure 7. Plot of: (a) $\triangle \mathrm{PESQ}$ where higher scores signify better speech quality, (b) $\triangle \mathrm{CD}$ where lower values signify better speech quality, (c) $\triangle \mathrm{RDT}$ where lower values signify better dereverberation, and (d) $\Delta$ STOI where higher scores signify better intelligibility. The graphs are against the index of the acoustic environment and, hence, against the $T_{60}$ and the DRR. The mean over the noises of white, babble and factory at $20 \mathrm{~dB}$ RSNR is shown.

the algorithm has a $\triangle \mathrm{PESQ}$ of 0.35 and 0.25 , respectively, for the RSNR of $20 \mathrm{~dB}$ averaged over the examined noises.

From Fig. 7(b), in terms of $\mathrm{CD}$, the KF algorithm yields improved speech quality in acoustic environments with a $T_{60}$ from 0.18 to $1.05 \mathrm{~s}$. The KF algorithm shows a deteriorating CD improvement with increasing $T_{60}$. Compared to the unprocessed signal, for a $T_{60}$ of 0.3 and $1 \mathrm{~s}$, the algorithm has a CD improvement of approximately -1.1 and -0.6 , respectively, for $20 \mathrm{~dB}$ RSNR averaged over the tested noise types.

From Fig. 7(c), we observe that the KF algorithm yields improved speech dereverberation in challenging acoustic conditions and that the $\triangle \mathrm{RDT}$ improves with increasing $T_{60}$ values. From the raw RDT of noisy reverberant speech in Fig. 7(c), we observe that the indexes A and $\mathrm{J}$ have very low reverberation. The indexes A and J have raw RDT scores 0.4 and 0.7, respectively. The indexes G-I and T-V have high reverberation with a high raw RDT score. The proposed algorithm in these high reverberation cases achieves a large RDT improvement decreasing the RDT metric from approximately 2.6 to 0.8 .

From Fig. 7(d), the algorithm shows marginally improved STOI performance for the tested $T_{60}$ range compared to the unprocessed signal and to the examined baselines. For a $T_{60}$ of 0.3 and $1 \mathrm{~s}$, the algorithm has a $\Delta$ STOI of 0.03 and 0.07 , respectively. $\Delta$ STOI slightly increases with increasing $T_{60}$.

Figure 8 shows the same graphs as in Fig. 7 but for a RSNR of $10 \mathrm{~dB}$. For the examined $T_{60}$ values and for white, babble and factory noises, the KF algorithm shows a consistent improvement in the PESQ, CD, RDT and STOI metrics.

Figure 9 depicts the same set of graphs as in Figs. 7 and 8 but for a RSNR of $5 \mathrm{~dB}$. For all the examined RSNRs, the KF algorithm shows a consistent improvement in the examined metrics, depending on the $T_{60}$. From Fig. 9(a), we observe that 


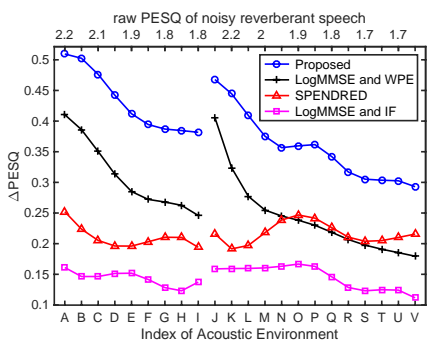

(a)

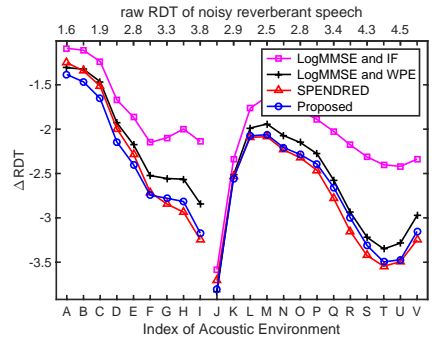

(c)

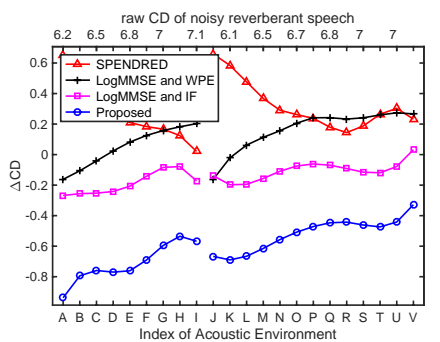

(b)

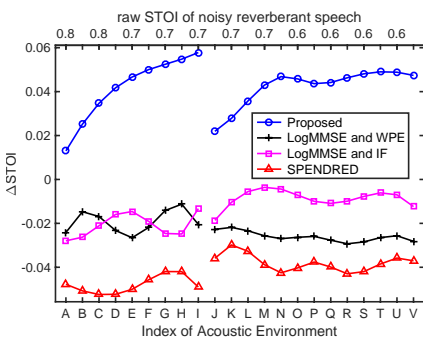

(d)
Figure 8. Plot of: (a) $\triangle \mathrm{PESQ}$, (b) $\triangle \mathrm{CD}$, (c) $\triangle \mathrm{RDT}$, and (d) $\triangle \mathrm{STOI}$. The graphs are against the index of the acoustic environment. The average over the noise types of white, babble and factory at $10 \mathrm{~dB}$ RSNR is shown.

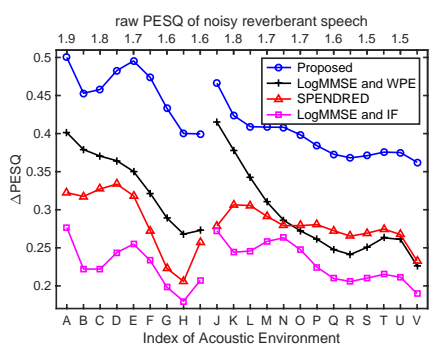

(a)

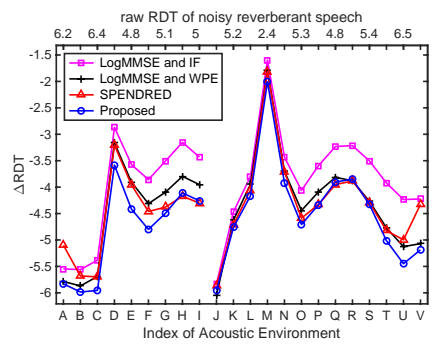

(c)

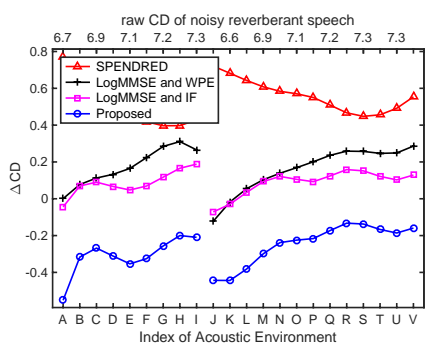

(b)

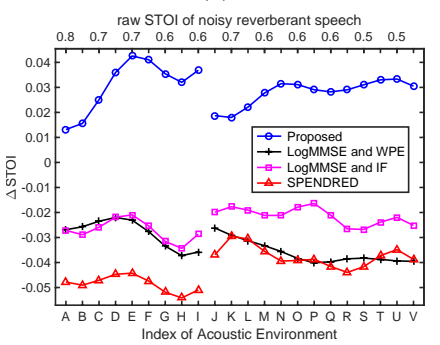

(d)
Figure 9. Plot of: (a) $\triangle \mathrm{PESQ}$, (b) $\triangle \mathrm{CD}$, (c) $\triangle \mathrm{RDT}$, and (d) $\triangle \mathrm{STOI}$. The graphs are against the index of the acoustic environment. The average over the noise types of white, babble and factory at $5 \mathrm{~dB}$ RSNR is shown.

the $\triangle \mathrm{PESQ}$ scores of the algorithm at $5 \mathrm{~dB}$ RSNR are similar to the $\triangle$ PESQ scores at $10 \mathrm{~dB}$ RSNR shown in Fig. 8(a).

The KF algorithm shows improved PESQ performance for the examined RSNRs from $5 \mathrm{~dB}$ to $20 \mathrm{~dB}$. The algorithm has better speech quality performance compared to the baselines for all the examined $T_{60}$ values. Compared to the unprocessed noisy reverberant speech, for the RSNR of $5 \mathrm{~dB}$, the algorithm has a $\triangle$ PESQ of about 0.45 for a $T_{60}$ of $0.6 \mathrm{~s}$ and a raw PESQ of 1.6. For the RSNR of $20 \mathrm{~dB}$, the algorithm has a $\triangle \mathrm{PESQ}$ of approximately 0.3 for a $T_{60}$ of $0.6 \mathrm{~s}$ and a raw PESQ of 2 . Comparing Figs. 7-9, we observe that the raw PESQ decreases with decreasing RSNR while the $\triangle \mathrm{PESQ}$ scores increase.

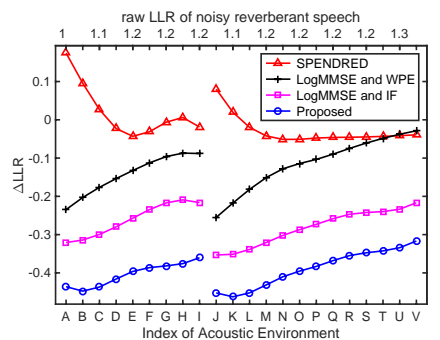

(a)

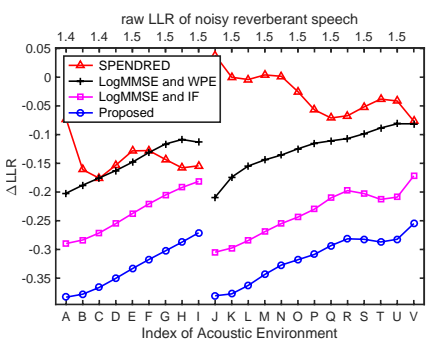

(b)
Figure 10. Plot of $\triangle$ LLR where lower scores signify better speech quality when white noise is used: (a) at $20 \mathrm{~dB}$ RSNR, and (b) at $10 \mathrm{~dB}$ RSNR.

The KF algorithm yields improved dereverberation performance in terms of RDT in adverse conditions in Fig. 9(c). The algorithm's performance improves with decreasing RSNR. We observe that the indexes A-C and J-K have a high raw RDT despite that they have low reverberation at $5 \mathrm{~dB}$ RSNR, when the effect of noise is higher than that of reverberation.

Figure 10 shows the log-likelihood ratio (LLR) when white noise is used at 20 and $10 \mathrm{~dB}$ RSNR. Decreasing the RSNR from 20 to $10 \mathrm{~dB}$ increases the raw LLR of noisy reverberant speech and deteriorates the $\triangle$ LLR. The results of LLR [87], which has been used to evaluate the dereverberation algorithm in [86], resemble the results of CD. Both CD and LLR have been used in [4]. It was found in [88] that LLR correlates well with speech quality although slightly less well than PESQ.

\section{B. Overall Performance Against the RSNR}

This section investigates the performance of the algorithm against the RSNR. If the reverberation is counted as additional noise, the overall SNR is always less than both the RSNR and the DRR. Figure 11 presents the algorithm's performance compared to the tested baselines for RSNRs from 0 to $20 \mathrm{~dB}$. Figures 11(a) and 11(b) depict the $\triangle \mathrm{PESQ}$ for white noise when: (a) $T_{60}=0.40 \mathrm{~s}$ and $\mathrm{DRR}=0.17 \mathrm{~dB}$, which is case $\mathrm{L}$ in Table III, and (b) $T_{60}=0.73 \mathrm{~s}$ and DRR $=-2.1 \mathrm{~dB}$, which is case $R$. The indexes $L$ and $R$ were chosen because they have different $T_{60}$ and DRR values. The ordering of the legends in Fig. 11 matches that of the algorithms at high RSNRs.

Figures 11(c) and 11(d) illustrate the $\triangle$ PESQ against the RSNR, from 0 to $20 \mathrm{~dB}$, for babble and factory noises, for cases $\mathrm{L}$ and $\mathrm{R}$ in Table III respectively, for the KF algorithm compared to the baselines. Babble noise is used for the solid lines, the top axis and the legends; factory noise is used for the dashed lines. The $\triangle$ PESQ depends on the RSNR and the noise type and is, most of the times, increasing with decreasing RSNRs. Compared to the unprocessed speech in Fig. 11(c), the algorithm's $\triangle \mathrm{PESQ}$ is approximately 0.35 for factory noise for high RSNRs, while it is decreasing for lower RSNRs.

Figure 12 examines the RDT improvement, $\triangle \mathrm{RDT}$, against the RSNR, from 0 to $20 \mathrm{~dB}$, for white noise, for cases $\mathrm{L}$ and $\mathrm{R}$ in Table III, in (a) and (b). Figure 12 depicts the $\Delta$ RDT against the RSNR for babble and factory noises, for cases L and R, in (c) and (d). Babble noise is used for the solid lines, the top axis and the legends while factory noise is used for the dashed lines. Figures 12(a)-(d) show that the $\Delta$ RDT improves and the raw 


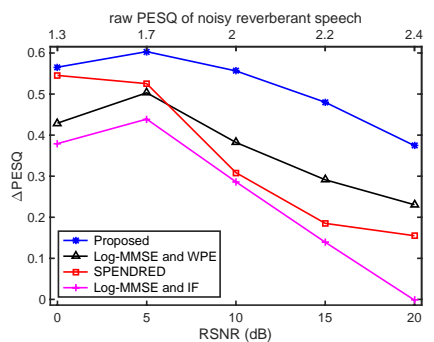

(a)

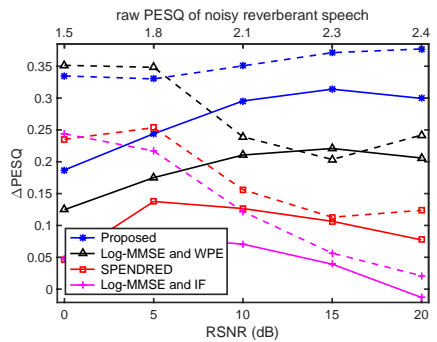

(c)

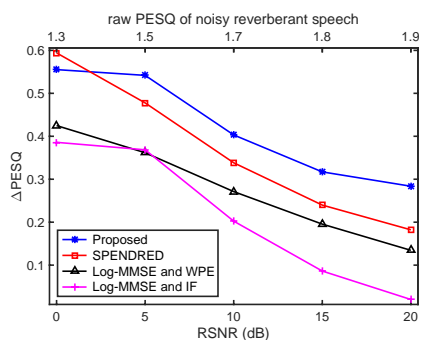

(b)

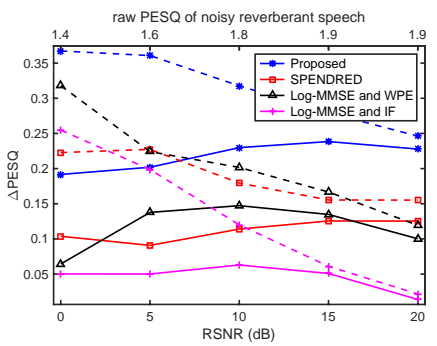

(d)
Figure 11. Plot of $\triangle$ PESQ against RSNR for: (a), (b) white noise, and (c) (d) babble noise (solid lines) and factory noise (dashed lines). In (a) and (c) $T_{60}=0.40 \mathrm{~s}$ and DRR $=0.17 \mathrm{~dB}$ (i.e. index $\mathrm{L}$ in Table III). In (b) and (d) $T_{60}=0.73 \mathrm{~s}$ and DRR $=-2.1 \mathrm{~dB}$ (i.e. index R). In (c) and (d), babble noise is used for the top axis and for the legends.

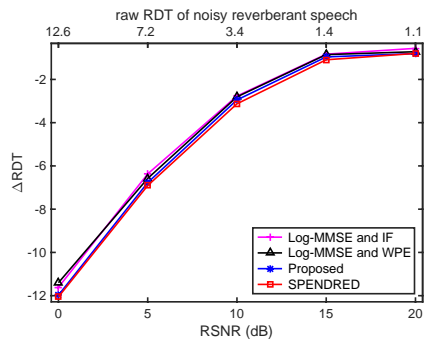

(a)

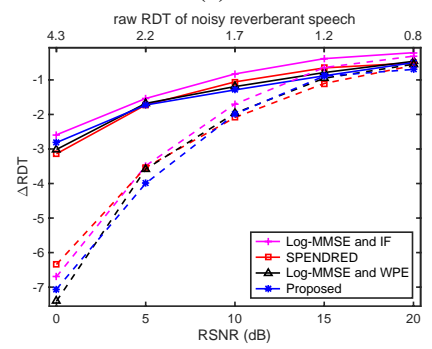

(c)

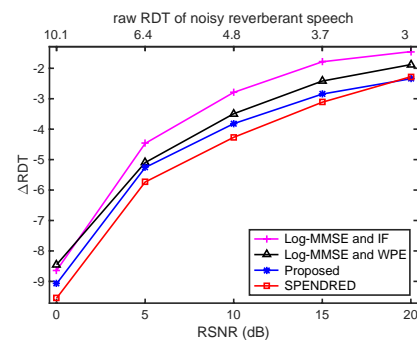

(b)

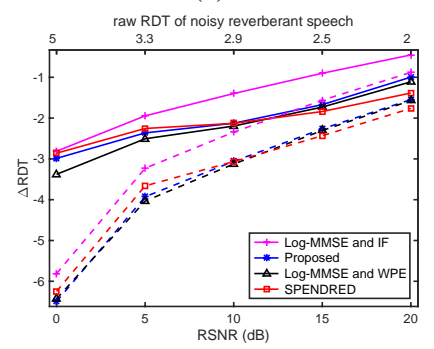

(d)
Figure 12. Plot of $\triangle$ RDT against RSNR for: (a), (b) white noise, and (c), (d) babble noise (solid lines) and factory noise (dashed lines). In (a) and (c), $T_{60}=0.40 \mathrm{~s}$ and DRR $=0.17 \mathrm{~dB}$ (i.e. index L in Table III). In (b) and (d), $T_{60}=0.73 \mathrm{~s}$ and DRR $=-2.1 \mathrm{~dB}$ (i.e. index R). In (c) and (d), babble noise is used for the top axis and for the legends.

RDT of noisy reverberant speech increases with decreasing RSNR. Also, the $\triangle$ RDT scores are better for stationary white noise than for non-stationary factory and babble noises.

Figure 13 shows the $\triangle \mathrm{PESQ}$ and the $\triangle \mathrm{RDT}$ against the RSNR, from 5 to $20 \mathrm{~dB}$, for the phase-aware KF algorithm for white, factory and babble noises. Figure 13 examines the acoustic conditions $\mathrm{J}$ to $\mathrm{V}$ that correspond to the room $10 \times$ $7 \times 3 \mathrm{~m}$ in Table III. Figure 13 differs from Figs. 11 and 12 in presenting the indexes $\mathrm{J}$ to $\mathrm{V}$ and not only $\mathrm{L}$ and $\mathrm{R}$. According

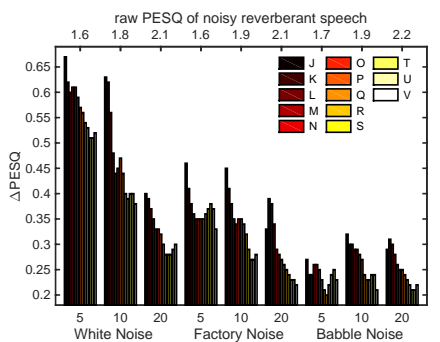

(a)

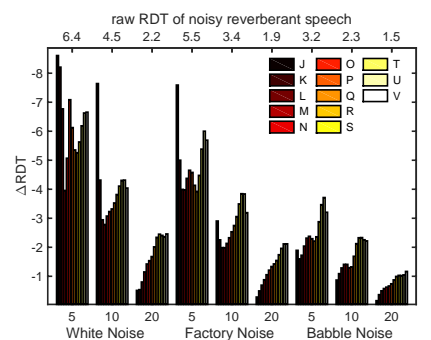

(b)
Figure 13. Plot of (a) $\triangle \mathrm{PESQ}$, and (b) $\triangle \mathrm{RDT}$ against RSNR (dB) for the algorithm for white, factory and babble noises. The indexes $\mathrm{J}$ to $\mathrm{V}$ are shown.

to Fig. 13(a), the $\triangle$ PESQ decreases as the $T_{60}$ increases. The $\triangle \mathrm{PESQ}$ increases with decreasing RSNR and the $\triangle \mathrm{PESQ}$ is higher for white noise than for factory and babble noises. In Fig. 13(b), the $\triangle$ RDT improves as the $T_{60}$ increases for high RSNRs and the $\triangle$ RDT improves with decreasing RSNR.

In summary, we have tested the KF algorithm in different noises, RSNRs and acoustic conditions with different $T_{60}$ and DRR. The algorithm is effective in decomposing noisy reverberant speech into speech, reverberation and noise. According to Figs. 7-13, the algorithm achieves a significant performance gain compared to the distorted speech and the baselines.

\section{Algorithmic Latency and Computational Complexity}

The latency of the phase-aware algorithm is $24 \mathrm{~ms}$ due to the AR modelling and the reverberation priors, using the parameters in Sec. IV. Using MATLAB on a laptop equipped with a $2.9 \mathrm{GHz}$ Intel Core i5 processor and parallel computation with 2 cores for processing STFT bins in parallel, the real-time factor from [89] is 35 . The complexity is high since $s_{t}, r_{t}$ and $n_{t}$ are estimated along with $\gamma_{t}$ and $\beta_{t}$ using (23)-(31) and Table II. The computation time is dominated by the calculation of the integrals over $u, u^{\prime}$ and $u^{\prime \prime}$ that use MATLAB's "integral" function. The real-time factor could be substantially reduced if sigma points were used to perform these integrals. The large number of parameters evaluated at every frame makes the realtime implementation of the $\mathrm{KF}$ algorithm that models interframe correlations more computationally challenging [52].

\section{CONCLUSION}

In this paper, we present a non-linear KF algorithm in the log-spectral domain to blindly suppress noise and reverberation while accounting for inter-frame dynamics. The first two moments of the posterior of the speech log-spectrum are estimated in noisy reverberant environments using a model that adaptively updates the reverberation parameters. The algorithm tracks the $\gamma_{t}$ and $\beta_{t}$ reverberation parameters to further improve speech estimation. We show by means of theoretical and experimental analyses that Kalman filtering can be performed in adverse conditions by performing the signal decompositions into speech, reverberation and noise, propagating backwards through the proposed signal model. Experimental results using instrumental measures show improved performance against the $T_{60}$ and the RSNR compared to the noisy reverberant speech and to alternative competing techniques that perform denoising and dereverberation either in concatenation or jointly. 


\section{REFERENCES}

[1] J. Eaton, N. D. Gaubitch, A. H. Moore and P. A. Naylor, "Estimation of room acoustic parameters: The ACE challenge," IEEE Trans. on Audio, Speech and Language Process., vol. 24, no. 10, pp. 1681-1693, Oct. 2016.

[2] J. Li and L. Deng and R. Haeb-Umbach and Y. Gong, Robust automatic speech recognition: A bridge to practical applications, Ch. 9: Reverberant speech recognition. Elsevier, ISBN: 978-0-12-802398-3, 2016.

[3] K. Kinoshita, M. Delcroix, S. Gannot, E. Habets et al., "A summary of the REVERB challenge: State-of-the-art and remaining challenges in reverberant speech processing research," EURASIP J. Adv. Signal Process., vol. 7, pp. 1-19, 2016

4] K. Kinoshita, M. Delcroix, T. Yoshioka, T. Nakatani, E. Habets et al., "The REVERB challenge: A common evaluation framework for dereverberation and recognition of reverberant speech," in Proc. IEEE Workshop on Applications of Signal Processing to Audio and Acoustics, New Paltz, NY, Oct. 2013

[5] C. S. J. Doire, M. Brookes, P. A. Naylor, D. Betts, C. M. Hicks, M. A. Dmour and S. H. Jensen, "Single-channel blind estimation of reverberation parameters," in Proc. IEEE Intl. Conf. on Acoustics, Speech and Signal Processing, Brisbane, Australia, pp. 31-35, April 2015.

[6] K. Lebart, J. M. Boucher, and P. N. Denbigh, "A New Method Based on Spectral Subtraction for Speech Dereverberation," Acta Acoustica vol. 87, pp. 359-366, 2001.

[7] S. O. Sadjadi and J. H. L. Hansen, "Blind spectral weighting for robust speaker identification under reverberation mismatch," IEEE Trans. on Audio, Speech and Language Process., vol. 22, no. 5, pp. 937-945, May 2014.

[8] I. J. Tashev, Sound Capture and Processing, Ch. 8: De-reverberation. John Wiley and Sons, ISBN: 978-0-470-31983-3, Aug. 2009.

[9] E. A. P. Habets, "Single- and Multi-Microphone Speech Dereverberation using Spectral Enhancement, Ch. 6: Late Reverberant Spectral Variance Estimation," Ph.D. dissertation, Universiteit Eindhoven, 2007.

10] Y. Wang and M. Brookes, "Model-based speech enhancement in the modulation domain," IEEE Trans. on Audio, Speech and Language Process., vol. 26, no. 3, pp. 580-594, March 2018.

[11] Y. Wang and M. Brookes, "Speech enhancement using an MMSE spectral amplitude estimator based on a modulation domain Kalman filter with a Gamma prior," in Proc. IEEE Int. Conf. Acoustics, Speech and Signal Process., March 2016.

[12] S. Braun, B. Schwartz, S. Gannot and E. A. P. Habets, "Late reverberation PSD estimation for single-channel dereverberation using relative convolutive transfer functions," in Proc. IEEE Intl. Workshop on Acoustic Signal Enhancement, Xi'an, China, Sept. 2016.

[13] A. Maezawa, K. Itoyama, K. Yoshii and H. G. Okuno, "Nonparametric Bayesian dereverberation of power spectrograms based on infinite-order autoregressive processes," IEEE Trans. on Audio, Speech and Language Process., vol. 22, no. 12, pp. 1918-1930, Dec. 2014

[14] M. Parchami, W.-P. Zhu and B. Champagne, "Model-based estimation of late reverberant spectral variance using modified weighted prediction error method," Speech Communication, vol. 92, pp. 100-113, 2017

[15] E. A. P. Habets, S. Gannot and I. Cohen, "Late reverberant spectral variance estimation based on a statistical model," IEEE Signal Processing Letters, vol. 16, no. 9, pp. 770-773, Sept. 2009.

[16] J. Moorer, "About this reverberation business," Computer Music Journal, vol. 3, no. 2, pp. 13-28, June 1979.

[17] J.-D. Polack, "La transmission de l'énergie sonore dans les salles," $\mathrm{Ph} . \mathrm{D}$ dissertation, Université du Maine, Le Mans, France, 1988.

[18] J. A. Moorer, "The optimum comb method of pitch period analysis of: Continuous digitized speech," IEEE ASSP, vol. 22, no. 5, pp. 330-338, Oct. 1974.

[19] M. Wölfel, "Enhanced speech features by single-channel joint compensation of noise and reverberation," IEEE Trans. on Audio, Speech and Language Process., vol. 17, no. 2, pp. 312-323, Feb. 2009.

[20] C. S. J. Doire, M. Brookes, P. A. Naylor et al, "Single-channel online enhancement of speech corrupted by reverberation and noise," IEEE Trans. on Audio, Speech and Language Process., vol. 25, no. 3, pp. 572-587, March 2017.

[21] C. S. J. Doire, "Single-channel enhancement of speech corrupted by reverberation and noise, Ch. 4: Single-channel enhancement of speech,' $\mathrm{Ph} . \mathrm{D}$. dissertation, Imperial College London, 2016.

[22] V. Leutnant, A. Krueger and R. Haeb-Umbach, "A new observation model in the logarithmic Mel power spectral domain for the automatic recognition of noisy reverberant speech," IEEE Trans. on Audio, Speech and Language Process., vol. 22, no. 1, pp. 95-109, Jan. 2014.
[23] V. Leutnant, "Bayesian estimation employing a phase-sensitive observation model for noise and reverberation robust automatic speech recognition, Ch. 4: Bayesian estimation of the speech feature posterior,' $\mathrm{Ph} . \mathrm{D}$. dissertation, Paderborn University, 2015.

[24] S. Braun and E. A. P. Habets, "Linear prediction based online dereverberation and noise reduction using alternating Kalman filters," IEEE Trans. on Audio, Speech, and Language Process., vol. 26, no. 6, pp. 1119-1129, June 2018 .

[25] S. Braun, "Speech dereverberation in noisy environments using timefrequency domain signal models, Ch. 4: Single-channel late reverberation PSD estimation," Ph.D. dissertation, University of ErlangenNuremberg, International Audio Laboratories Erlangen, 2018.

[26] V. Leutnant, A. Krueger and R. Haeb-Umbach, "Investigations into a statistical observation model for logarithmic Mel power spectral density features of noisy reverberant speech," in Proc. Speech Communication 10. ITG Symposium, Braunschweig, Germany, Sept. 2012.

[27] V. Leutnant and R. Haeb-Umbach, "An analytic derivation of a phasesensitive observation model for noise-robust speech recognition," in Proc. Conf. Int. Speech Communication Association, pp. 2395-2398, Brighton, Sept. 2009.

[28] T. Yoshioka, A. Sehr, M. Delcroix, K. Kinoshita, R. Maas, T. Nakatani, et al., "Making machines understand us in reverberant rooms: robustness against reverberation for automatic speech recognition," IEEE Signal Process., vol. 29, no. 6, pp. 114-126, 2012.

[29] M. Parchami, W.-P. Zhu and B. Champagne, "Speech dereverberation using weighted prediction error with correlated inter-frame speech components," Speech Communication, vol. 87, pp. 49-57, 2017.

[30] M. Delcroix, T. Yoshioka, A. Ogawa et al., "Linear prediction-based dereverberation with advanced speech enhancement and recognition technologies for the REVERB challenge," REVERB'14, 2014.

[31] M. Delcroix, T. Yoshioka, A. Ogawa et al., "Strategies for distant speech recognition in reverberant environments," EURASIP Journal on Advances in Signal Processing, vol. 60, July 2015.

[32] K. Kinoshita, M. Delcroix, H. Kwon, T. Mori, T. Nakatani, "Neural network-based spectrum estimation for online WPE dereverberation," in Proc. Conf. Int. Speech Communication Association, Stockholm, 2017.

[33] Z. Zhang, J. Geiger, J. Pohjalainen, A. E.-D. Mousa, W. Jin, and B. Schuller, "Deep learning for environmentally robust speech recognition," ACM Transactions on Intelligent Systems and Technology, vol. 9 no. 5, pp. 1-28, apr 2018

[34] Y. Xu, J. Du, L. R. Dai and C. H. Lee, "A regression approach to speech enhancement based on deep neural networks," IEEE Trans. on Audio, Speech and Language Process., vol. 23, no. 1, pp. 7-19, Jan. 2015.

[35] Y. Wang and D. Wang, "Towards scaling up classification-based speech separation," IEEE Trans. on Audio, Speech, and Language Process., vol. 21, no. 7, pp. 1381-1390, July 2013.

[36] H. Erdogan, J. R. Hershey, S. Watanabe, and J. L. Roux, "Phasesensitive and recognition-boosted speech separation using deep recurrent neural networks," in Proc. IEEE Int. Conf. Acoustics, Speech and Signal Process., Apr. 2015, pp. 708-712.

[37] Ephraim, Y. and Malah, D., "Speech enhancement using a minimum mean-square error log-spectral amplitude estimator," IEEE Trans. on Acoustics, Speech and Signal Process., vol. 33, no. 2, pp. 443-445, April 1985.

[38] K. Han, Y. Wang, D. Wang, W. S. Woods, I. Merks and T. Zhang, "Learning spectral mapping for speech dereverberation and denoising," IEEE Trans. on Audio, Speech and Language Process., vol. 23, no. 6, pp. 982-992, June 2015

[39] D. S. Williamson and D. Wang, "Time-frequency masking in the complex domain for speech dereverberation and denoising," IEEE Trans. on Audio, Speech and Language Process., vol. 25, no. 7, pp. 1492-1501, July 2017.

[40] Y. Zhao, Z.-Q. Wang, and D. Wang, "Two-stage deep learning for noisyreverberant speech enhancement," IEEE/ACM Transactions on Audio, Speech, and Language Processing, vol. 27, no. 1, pp. 53-62, jan 2019.

[41] Y. Sun, W. Wang, J. Chambers, and S. M. Naqvi, "Two-stage monaural source separation in reverberant room environments using deep neural networks,' IEEE/ACM Transactions on Audio, Speech, and Language Processing, vol. 27, no. 1, pp. 125-139, jan 2019.

[42] H. Erdogan, J. R. Hershey, S. Watanabe and J. Le Roux, "Deep Recurrent Networks for Separation and Recognition of Single-Channel Speech in Nonstationary Background Audio," in New Era for Robust Speech Recognition: Exploiting Deep Learning, S. Watanabe, M. Delcroix, F Metze and J. R. Hershey, Ed. Springer, 2017, ch. 7, pp. 165-186.

[43] F. Xiong, B. T. Meyer, B. Cauchi, A. Jukic, S. Doclo and S. Goetze, "Performance comparison of real-time single-channel speech derever- 
beration algorithms," in Proc. IEEE Int. Work. Hands-free Speech Communication and Microphone Arrays, San Francisco, March 2017.

[44] M. Kolbaek, Z.-H. Tan, and J. Jensen, "Speech intelligibility potential of general and specialized deep neural network based speech enhancement systems," IEEE/ACM Transactions on Audio, Speech, and Language Processing, vol. 25, no. 1, pp. 153-167, jan 2017.

[45] A. Kumar and D. Florencio, "Speech enhancement in multiple-noise conditions using deep neural networks," in Proc Interspeech Conf, 2016.

[46] R. Rehr and T. Gerkmann, "Normalized features for improving the generalization of DNN based speech enhancement," arXiv, 2018, [Online] Available: https://arxiv.org/pdf/1709.02175.pdf.

[47] K. Paliwal and A. Basu, "A speech enhancement method based on Kalman filtering," in Proc. IEEE Int. Conf. Acoustics, Speech and Signal Process., vol. 12, 1987, pp. 177-180.

[48] S. Gannot, D. Burshtein, and E. Weinstein, "Iterative and sequential Kalman filter-based speech enhancement algorithms," IEEE Trans. Speech Audio Processing, vol. 6, no. 4, pp. 373-385, Jul. 1998

[49] N. Ma, M. Bouchard, and R. A. Goubran, "Speech enhancemen using a masking threshold constrained Kalman filter and its heuristic implementations," IEEE Trans Audio, Speech, Language Processing, vol. 14, no. 1, pp. 19-32, 2006.

[50] S. So and K. K. Paliwal, "Modulation-domain Kalman filtering for single-channel speech enhancement," Speech Communication, vol. 53 no. 6, pp. $818-829$, July 2011

[51] S. So, K. K. Wójcicki, and K. K. Paliwal, "Single-channel speech enhancement using Kalman filtering in the modulation domain," in Proc. Conf. Int. Speech Communication Association, Makuhari, Sept. 2010.

[52] N. Dionelis and M. Brookes, "Phase-aware single-channel speech enhancement with modulation-domain Kalman filtering," IEEE Trans. on Audio, Speech and Language Process., vol. 26, no. 5, pp. 937-950, May 2018.

[53] N. Dionelis and M. Brookes, "Modulation-domain speech enhancement using a Kalman filter with a Bayesian update of speech and noise in the log-spectral domain," in Proc. IEEE Int. Work. Hands-free Speech Communication and Microphone Arrays, San Francisco, March 2017.

[54] Y. Wang, "Speech enhancement in the modulation domain, Ch. 5: Model-based speech enhancement in the modulation domain," Ph.D. dissertation, Imperial College London, 2015.

[55] B. Schwartz, S. Gannot, and E. A. P. Habets, "Online speech dereverberation using Kalman filter and EM algorithm," and Language Processing IEEE/ACM Transactions on Audio, Speech, vol. 23, no. 2, pp. 394-406, Feb. 2015.

[56] N. Dionelis, "On single-channel speech enhancement and on non-linear modulation-domain Kalman filtering," arXiv:1811.00078 [cs.SD], Nov. 2018, [Online] Available: https://arxiv.org/pdf/1811.00078.pdf.

[57] N. Dionelis and M. Brookes, "Speech enhancement using modulationdomain Kalman filtering with active speech level normalized logspectrum global priors," in Proc. European Signal Process. Conf., Kos, Aug. 2017

[58] N. Dionelis and M. Brookes, "Speech enhancement using Kalman filtering in the logarithmic Bark power spectral domain," in Proc. European Signal Process. Conf., Rome, Sept. 2018.

[59] S. J. Julier and J. K. Uhlmann, "Unscented filtering and nonlinear estimation," Proceedings of the IEEE, vol. 92, no. 3, pp. 401-422, 2004

[60] M. Brookes, "The Matrix Reference Manual, Stochastic Matrices," Imperial College London, 2018, [Online] Available: http://www.ee.ic.ac.uk/hp/staff/dmb/matrix/expect.html

[61] T. de M. Prego, A. A. de Lima, R. Zambrano-Lopez and S. L. Netto, "Blind estimators for reverberation time and direct-to-reverberant energy ratio using subband speech decomposition," in Proc. IEEE Workshop on Applications of Signal Processing to Audio and Acoustics, New Paltz, $N Y$, Oct. 2015.

[62] T. de M. Prego, A. A. de Lima and S. L. Netto, "A blind algorithm for reverberation-time estimation using subband decomposition of speech signals," Journal of the Acoustical Society of America, vol. 131, no. 4 , pp. 2811-2816, April 2012.

[63] J. Eaton, N. D. Gaubitch and P. A. Naylor, "Noise-robust reverberation time estimation using spectral decay distributions with reduced computational cost," in Proc. IEEE Int. Conf. on Acoustics, Speech and Signal Process., Vancouver, May 2013

[64] J. Y. C. Wen, E. A. P. Habets and P. A. Naylor, "Blind estimation of reverberation time based on the distribution of signal decay rates," in Proc. IEEE Intl. Conf. on Acoustics, Speech and Signal Processing, Las Vegas, April 2008

[65] S. Theodoridis, Machine Learning: A Bayesian and Optimization Perspective, Ch. 3.10.1: Linear Regression: The nonwhite Gaussian noise case. Elsevier, ISBN: 978-0-12-801522-3, 2015.
[66] S. J. Russell and P. Norvig, Artificial Intelligence: a Modern Approach Ch. 15: Probabilistic Reasoning over Time. Prentice Hall Pearson Education International, Second Edition, ISBN: 0-13-080302-2, 2003.

[67] R. Chen, C.-F. Chan and H. C. So, "Model-based speech enhancement with improved spectral envelope estimation via dynamics tracking," IEEE Trans. on Audio, Speech, and Language Process., vol. 20, no. 4, pp. 1324-1336, May 2012.

[68] H. Gamper and I. J. Tashev, "Blind reverberation time estimation using a convolutional neural network," in Proc. International Workshop on Acoustic Signal Enhancement (IWAENC), Tokyo, Japan, Sept. 2018.

[69] T. Nakatani, T. Yoshioka, K. Kinoshita, M. Miyoshi, and B.-H. Juang, "Speech dereverberation based on variance-normalized delayed linear prediction," IEEE Trans. on Audio, Speech and Language Process., vol. 18, no. 7, pp. 1717-1731, Sept. 2010.

[70] M. Delcroix, T. Yoshioka et al., "Linear prediction-based dereverberation with advanced speech enhancement and recognition technologies for the REVERB challenge," in Proc. REVERB Workshop 2014, 2014.

[71] S. So, K. K. Wocicki, J. G. Lyons, A. P. Stark, and K. K. Paliwal, "Kalman filter with phase spectrum compensation algorithm for speech enhancement," in Proc. IEEE Int. Conf. Acoustics, Speech and Signal Process., 2009, pp. 4405-4408.

[72] T. Gerkmann and R. C. Hendriks, "Unbiased MMSE-based noise power estimation with low complexity and low tracking delay," IEEE Trans. on Audio, Speech, and Language Process., vol. 20, no. 4, pp. 1383-1393, 2012.

[73] M. Gales and S. Young, "Cepstral parameter compensation for HMM recognition in noise," Speech Communication, vol. 12, no. 3, pp. 231 239, July 1993.

[74] R. F. Astudillo, "Integration of short-time Fourier domain speech enhancement and observation uncertainty techniques for robust automatic speech recognition, Ch. 5: Fourier domain uncertainty models," Ph.D. dissertation, Technical University of Berlin, 2010.

[75] M. Brookes, "VOICEBOX: A speech processing toolbox for MATLAB," Imperial College London, Software Library, 2018, [Online] Available: http://www.ee.imperial.ac.uk/hp/staff/dmb/voicebox/voicebox.html.

[76] J. B. Allen and D. A. Berkley, "Image method for efficiently simulating small-room acoustics," J. Acoust. Soc. Amer., vol. 65, no. 4, pp. 943-950, Apr 1979

[77] E. De Sena, N. Antonello, M. Moonen and T. van Waterschoot, "On the modeling of rectangular geometries in room acoustic simulations," IEEE Trans. on Audio, Speech and Language Process., vol. 23, no. 4, pp. 774-786, April 2015.

[78] ITU-T, "Perceptual evaluation of speech quality (PESQ), an objective method for end-to-end speech quality assessment of narrowband telephone networks and speech codecs," ITU-T Rec P.862, Feb. 2001

[79] N. Kitawaki, H. Nagabuchi, and K. Itoh, "Objective quality evaluation for low bit-rate speech coding systems," IEEE Journal on Selected Areas in Communications, vol. 6, no. 2, pp. 262-273, 1988.

[80] J. Y. C. Wen and P. A. Naylor, "An evaluation measure for reverberan speech using decay tail modelling," in Proc. European Signal Process. Conf., Florence, pp. 1-5, Sept. 2006.

[81] C. H. Taal, R. C. Hendriks, R. Heusdens and J. Jensen, "An algorithm for intelligibility prediction of time-frequency weighted noisy speech," IEEE Trans. on Audio, Speech and Language Process., vol. 19, no. 7, pp. 2125-2136, Sept. 2011

[82] C. Taal, "MATLAB code for algorithms," Software Package, 2011-2015, [Online] Available: http://www.ceestaal.nl/matlab-code/.

[83] J. Garofolo, L. Lamel, W. Fisher et al, "TIMIT acoustic-phonetic continuous speech corpus," Corpus LDC93S1, Linguistic Data Consortium, Philadelphia, 1993.

[84] H. Steeneken and F. Geurtsen, "Description of the RSG-10 noise database," TNO Institute for perception, Tech. Rep. IZF 1988-3, 1988.

[85] E. Habets, "Room impulse response generator," April 2016, [Online] Available: https://github.com/ehabets/RIR-Generator.

[86] D. Schmid, G. Enzner, S. Malik, D. Kolossa and R. Martin, "Variational Bayesian inference for multichannel dereverberation and noise reduction," IEEE Trans. on Audio, Speech and Language Process., vol. 22, no. 8, pp. 1320-1335, 2011

[87] S. R. Quackenbush, T. P. Barnwell, III, and M. A. Clements, Objective Measures of Speech Quality. Prentice Hall, Jan. 1988.

[88] Y. Hu and P. C. Loizou, "Evaluation of objective quality measures for speech enhancement," IEEE Trans. on Audio, Speech and Language Process., vol. 16, no. 1, pp. 229-238, Jan. 2008.

[89] N. D. Gaubitch, H. W. Lollmann, M. Jeub, T. H. Falk, P. A. Naylor, P. Vary and M. Brookes, "Performance comparison of algorithms for blind reverberation time estimation from speech," in Proc. IEEE Int. Work. Acoustic Signal Enhancement, Sept. 2012 